\title{
RESIDUAL BASED A POSTERIORI ERROR ESTIMATORS FOR EDDY CURRENT COMPUTATION
}

\author{
Rudi Beck ${ }^{1}$, Ralf Hiptmair ${ }^{2}$, Ronald H.W. Hoppe ${ }^{3}$ And Barbara Wohlmuth ${ }^{3}$
}

\begin{abstract}
We consider $\boldsymbol{H}(\mathbf{c u r l} ; \Omega)$-elliptic problems that have been discretized by means of Nédélec's edge elements on tetrahedral meshes. Such problems occur in the numerical computation of eddy currents. From the defect equation we derive localized expressions that can be used as a posteriori error estimators to control adaptive refinement. Under certain assumptions on material parameters and computational domains, we derive local lower bounds and a global upper bound for the total error measured in the energy norm. The fundamental tool in the numerical analysis is a Helmholtz-type decomposition of the error into an irrotational part and a weakly solenoidal part.
\end{abstract}

Résumé. Nous considérons des estimateurs d'erreur a posteriori efficaces et fiables pour l'approximation des champs électromagnetiques par la méthode des éléments finis curl-conformes. En particulier, en utilisant les éléments à arêtes de Nédélec sur des maillages tétrahédraux, nous dériverons des bornes inférieures locales et une borne supérieure globale pour l'erreur totale mesurée à la norme d'espace $\boldsymbol{H}(\operatorname{curl} ; \Omega)$. Le moyen fondamental en analyse numérique est une décomposition d'Helmholtz de l'erreur en une part irrotative et une part faiblement solenoïdale.

Mathematics Subject Classification. 65N15, 65N30, 65N50.

Received: March 30, 1999.

\section{INTRODUCTION}

The computation of quasistatic electromagnetic fields in conductors usually employs the eddy current model $[2,5,23,33]$. For the transient case, if we use formulations based on the electric field, we end up with the degenerate parabolic initial-boundary value problem

$$
\begin{aligned}
\partial_{t}(\sigma \mathbf{E})+\operatorname{curl} \chi \operatorname{curl} \mathbf{E} & =-\partial_{t} \mathbf{j} & & \text { in } \Omega \\
\mathbf{E} \times \mathbf{n} & =0 & & \text { on } \Gamma:=\partial \Omega \\
\mathbf{E}(., 0) & =\mathbf{E}_{0} & & \text { in } \Omega .
\end{aligned}
$$

Here the unknown quantity is the electric field $\mathbf{E}: \Omega \times[0, T] \mapsto \mathbb{R}^{3}$ and $\Omega \subset \mathbb{R}^{3}$ stands for a connected bounded polyhedral computational domain. Though the equations are initially posed on the entire space $\mathbb{R}^{3}$,

Keywords and phrases. Residual based a posteriori error estimation, Nédélec's edge elements, Helmholtz decomposition, eddy currents.

1 ZIB-Berlin, Takustr. 7, 14195 Berlin, Germany. e-mail: beck@sc.zib-berlin.de

2 SFB 382, Universität Tübingen, Auf der Morgenstelle 10, 72076 Tübingen, Germany. e-mail: hiptmair@na.uni-tuebingen.de

3 Mathematisches Institut, Universität Augsburg, Universitätsstr. 14, 86159 Augsburg, Germany.

e-mail: hoppe@math.uni-augsburg.de; wohlmuth@math.uni-augsburg.de 


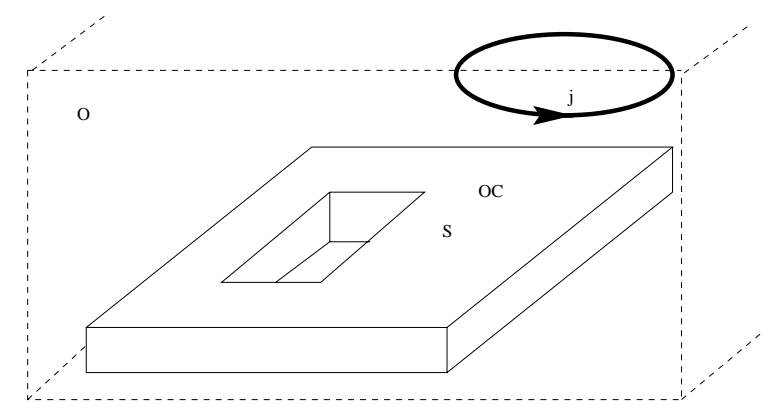

Figure 1. A model problem for eddy current computation (cf. Chap. 8 in [24]).

we can switch to a bounded domain by introducing an artificial boundary sufficiently removed from the region of interest. This is commonplace in engineering simulations [29].

Further, $\chi \in L^{\infty}(\Omega)$ denotes the bounded uniformly positive inverse of the magnetic permeability. We confine ourselves to linear isotropic media, i.e. $\chi$ is a scalar function of the spatial variable $\mathbf{x} \in \Omega$ only. Hence, for some $\underline{\chi}, \bar{\chi}>0$ holds $0<\underline{\chi} \leq \chi \leq \bar{\chi}$ a.e. in $\Omega$. We rule out anisotropy also for the conductivity $\sigma \in L^{\infty}(\Omega)$, for which holds $\sigma \geq 0$ a.e. in $\Omega$. Usually, there is a crisp distinction between conducting regions, where $\sigma$ is bounded away from zero, and insulating regions, where $\sigma=0$. We will take for granted that $\sigma \geq \underline{\sigma}>0$, for some bound $\underline{\sigma}>0$, wherever $\sigma \neq 0$. The right-hand side $\mathbf{j}=\mathbf{j}(\mathbf{x}, t)$ is a time-dependent vectorfield in $\boldsymbol{L}^{2}(\Omega)$, which represents the source current. For physical reasons $\operatorname{div} \mathbf{j}(t,)=$.0 a.e. in $\Omega$ and for all times. We remark that in many applications the exciting current, for instance the current in a coil, is provided through an analytic expression. A typical arrangement is depicted in Figure 1.

We remark that (1) is an ungauged formulation, as we have already dropped the divergence constraint $\operatorname{div} \mathbf{E}=0$. Obviously, this forfeits uniqueness of the solution in parts of the domain where $\sigma=0$. However, the only relevant quantity there is $\operatorname{curl} \mathbf{E}$, which remains unique. Where $\mathbf{E}$ is of interest, inside the conductor, we have $\sigma>0$. There $\mathbf{E}$ is unique and $\operatorname{div} \mathbf{E}=0$ is satisfied due to the solenoidality of the right-hand side.

For the sake of stability, timestepping schemes for (1) have to be L-stable [38]. This requirement can only be met by implicit schemes like SDIRK-methods. In each timestep they entail the solution of a degenerate elliptic boundary value problem of the form

$$
\begin{aligned}
& \operatorname{curl} \chi \operatorname{curl} \mathbf{u}+\beta \mathbf{u}=\mathbf{f} \quad \text { in } \Omega \\
& \mathbf{u} \times \mathbf{n}=0 \quad \text { on } \partial \Omega \text {. }
\end{aligned}
$$

In this context, $\mathbf{u}$ denotes the new approximation to $\mathbf{E}$ to be computed in the current timestep, and $\mathbf{f}$ depends on $\mathbf{j}$ and the approximation of $\mathbf{E}$ in the previous timestep. Note that we can still assume $\operatorname{div} \mathbf{f}=0$. The coefficient $\beta$ agrees with $\sigma$ except for a scaling by the length of the current timestep; accordingly, $0<\underline{\beta} \leq \beta \leq \bar{\beta}$ a.e. in $\Omega_{C}$.

Problem (2) cast in weak form yields a variational problem in the Hilbert space $\boldsymbol{H}(\mathbf{c u r l} ; \Omega)$ of $\boldsymbol{L}^{2}(\Omega)$ vectorfields whose curl is square integrable:

Find $\mathbf{u} \in \boldsymbol{H}_{0}(\mathbf{c u r l} ; \Omega)$ such that

$$
(\chi \operatorname{curl} \mathbf{u}, \operatorname{curl} \mathbf{q})_{L^{2}(\Omega)}+(\beta \mathbf{u}, \mathbf{q})_{\boldsymbol{L}^{2}(\Omega)}=(\mathbf{f}, \mathbf{q})_{L^{2}(\Omega)}, \quad \forall \mathbf{q} \in \boldsymbol{H}_{0}(\operatorname{curl} ; \Omega) .
$$

A subscript 0 indicates that vanishing tangential traces on $\partial \Omega$ are imposed on the fields (for details on traces see, e.g. $[4,37])$.

For $\beta$ uniformly positive a.e. in $\Omega$ the Lax-Milgram lemma guarantees existence and uniqueness of a solution of (3). If $\beta=0$ on sets of positive measure, we can still expect a unique solution in the quotient space $\boldsymbol{H}_{0}(\operatorname{curl} ; \Omega) / \operatorname{Ker}(\operatorname{curl})$. 
It is now generally accepted that an appropriate finite element discretization of (3) should rely on genuine $\boldsymbol{H}(\mathbf{c u r l} ; \Omega)$-conforming schemes that merely enforce the typical tangential continuity of the field across interelement boundaries [3,21,22]. For simplicial meshes $\boldsymbol{H}(\mathbf{c u r l} ; \Omega)$-conforming finite elements of arbitrary polynomial order were first introduced by Nédélec [49], generalizing the lowest order Whitney elements [56]. Similar schemes are also known for other shapes of elements [34,49]. For all of them standard a priori error bounds can be established $[47,48,52]$. Ultimately, the discretization of $(3)$ by these so-called edge elements leads to a large sparse system of equations for the degrees of freedom of the finite element space. Usually, an approximate solution can only be obtained by iterative methods $[28,39]$.

Denoting by $\mathbf{u}_{h}$ the exact solution of the discretized problem and by $\tilde{\mathbf{u}}_{h}$ some iterative approximation, we are interested in an efficient and reliable residual based a posteriori error estimator for the total error $\mathbf{e}:=\mathbf{u}-\tilde{\mathbf{u}}_{h}$ with respect to relevant norms. The most significant is the energy (semi)norm $\|\cdot\|_{\mathfrak{E} ; \Omega}$ related to problem (1) defined by

$$
\|\mathbf{u}\|_{\mathfrak{E} ; \Omega}^{2}:=(\chi \operatorname{curl} \mathbf{u}, \operatorname{curl} \mathbf{u})_{L^{2}(\Omega)}+(\beta \mathbf{u}, \mathbf{u})_{\mathbf{L}^{2}(\Omega)}, \quad \mathbf{u} \in \boldsymbol{H}_{0}(\operatorname{curl} ; \Omega)
$$

In the current context, local a posteriori error estimation serves two purposes. Firstly, the error estimator can be used for adaptive local refinement and coarsening of the underlying triangulation. Since the fields feature strong singularities at reentrant corners [31] and at irregular material interfaces [32], a higher resolution of the mesh in these zones is desirable. Precisely how much can only be concluded on the basis of information about the local error. Secondly, information about the accuracy of the finite element solution is also required to balance the spatial and temporal errors in the context of adaptive timestepping for the original parabolic problem $[17,18]$.

We note that a posteriori error estimators for adaptive local grid refinement are well established tools in the efficient numerical solution of elliptic boundary value problems. In the framework of standard conforming finite element approaches we acknowledge the pioneering work due to Babuska and Rheinboldt $[8,9]$ and the more recent articles $[11,12,35,36,54,57]$. Further references can be found in the survey article by Bornemann et al. [19] and in the excellent monography by Verfürth [55]. In the context of nonconforming techniques we mention $[41,43]$. For mixed finite element methods involving Raviart-Thomas elements we refer to $[1,25,26,42$, 44,45]. However, as far as finite element approximations based on Nédélec's curl-conforming edge elements are concerned, to the authors' knowledge no work on a posteriori error estimation has been done so far.

The paper is organized as follows. In Section 2, we will introduce the curl-conforming approximation of (3) by Nédélec's edge elements. In addition we are going to supply a few technical devices required for the proofs. Then, in Section 3, we consider the variational problem satisfied by the total error $\mathbf{e}$ and state the main result of this paper in terms of a cheaply computable, efficient and reliable a posteriori error estimator for $\|\mathbf{e}\|_{\mathfrak{E}: \Omega}$. As the main tool we will use a Helmholtz type decomposition of $\mathbf{e}$ into a curl-free part $\mathbf{e}^{0}$ and a " $\beta$-weakly solenoidal" part $\mathbf{e}^{\perp}$. In particular, Section 4 contains the estimation of the irrotational part $\mathbf{e}^{0}$ whereas Section 5 is devoted to the weakly solenoidal part $\mathbf{e}^{\perp}$. In both cases, the estimates result from an evaluation of the residuum with respect to a dual norm. In the final section we report on numerical experiments that examine the performance of the error estimator for a wide range of model problems.

\section{Finite ELEMENT SPACES}

We consider the finite element approximation of (3) by means of Nédélec's edge elements with respect to a hierarchy $\mathcal{T}_{h_{k}}, k \in \mathbb{N}_{0}$, of simplicial triangulations of $\Omega$ generated by successive local refinement of an initial coarse triangulation $\mathcal{T}_{h_{0}}$. We use the standard refinement process developed by Bank et al. [10,11] in the $2 \mathrm{D}$ case which has been extended to the 3D setting in $[16,50]$ (cf. also [19]). Alternative schemes are also available $[7,13,46]$. For a description of the refinement strategies we refer to the literature cited above.

We demand that the coarsest mesh $\mathcal{T}_{h_{0}}$ can resolve the boundaries of the conductors. This means that any element either entirely belongs to the conducting region or to the nonconducting region. 
We fix some $\mathcal{T}_{h_{k}}$ within the hierarchy and, for notational convenience, omit the lower index $k$, i.e., $\mathcal{T}_{h}:=\mathcal{T}_{h_{k}}$. For $D \subseteq \bar{\Omega}$, the sets of vertices, edges and faces in $D$ are denoted by $\mathcal{N}_{h}(D), \mathcal{E}_{h}(D)$ and $\mathcal{F}_{h}(D)$, respectively. If $D=\bar{\Omega}$, we will simply write $\mathcal{N}_{h}, \mathcal{E}_{h}, \mathcal{F}_{h}$ and refer to $\mathcal{N}_{h}^{\text {int }}, \mathcal{E}_{h}^{\text {int }}, \mathcal{F}_{h}^{\text {int }}$ and $\mathcal{N}_{h}^{\Gamma}, \mathcal{E}_{h}^{\Gamma}, \mathcal{F}_{h}^{\Gamma}$ as the sets of vertices, edges and faces located in the interior of $\Omega$ and on the boundary $\Gamma$, respectively. Those interior edges that belong to $\bar{\Omega}_{C}$ constitute the set $\mathcal{F}_{h}^{C}$ and for the set of elements in $\bar{\Omega}_{C}$ we write $\mathcal{T}_{h}^{C}$.

All the edges have to be endowed with a fixed internal orientation (direction). We denote by $h_{T}$ and $h_{F}$ the maximal diameter of an element $T \in \mathcal{T}_{h}$ and a face $F \in \mathcal{F}_{h}$. Since the refinement rules imply regularity and local quasiuniformity of the hierarchy of triangulations ( $c f .[16])$, there exist constants $\kappa_{1}>0$ and $\kappa_{2}>0$ depending only on the local geometry of the initial triangulation $\mathcal{T}_{0}:=\mathcal{T}_{h_{0}}$ such that

$$
\begin{aligned}
& h_{T^{\prime}} \leq \kappa_{1} h_{T} \quad \text { for } T, T^{\prime} \in \mathcal{T}_{h}, T \cap T^{\prime} \neq \emptyset \\
& h_{F} \leq \kappa_{2} h_{T} \quad \text { for } F \in \mathcal{F}_{h}(T) \text {. }
\end{aligned}
$$

Following Nédélec's construction of simplicial edge elements in [49], we denote by $\mathcal{P}_{k}(D), k \geq 0$, the linear space of multivariate polynomials of degree $\leq k$ on $D$, and refer to $\tilde{\mathcal{P}}_{k}(D), k \geq 0$, as the subspace of homogeneous polynomials of degree $k$. We define

$$
\mathcal{S}_{k}(D):=\left\{\mathbf{p} \in \tilde{\mathcal{P}}_{k}(D)^{3},\langle\mathbf{x}, \mathbf{p}\rangle:=\sum_{i=1}^{3} x_{i} p_{i}=0\right\}, \quad k \geq 1 .
$$

Then, for $T \in \mathcal{T}_{h}$ and $k \geq 1$, the local space for the Nédélec element is given by

$$
\mathcal{N D}_{k}(T):=\mathcal{P}_{k-1}(D)^{3} \oplus \mathcal{S}_{k}(D) .
$$

In the special case of lowest order edge elements, $k=1$, we find the representation

$$
\mathcal{N D}_{1}(T):=\left\{\mathbf{x} \mapsto \mathbf{a}+\mathbf{b} \times \mathbf{x}, \mathbf{a}, \mathbf{b} \in \mathbb{R}^{3}\right\} .
$$

Appropriate degrees of freedom are provided by linear functionals on $\mathcal{N} \mathcal{D}_{k}(T)$ of the form (cf. e.g. [49])

$$
\begin{aligned}
& \text { (i) } \quad \mathbf{q} \mapsto \int_{E}\langle\mathbf{q}, \mathbf{t}\rangle p \mathrm{~d} s, \quad p \in \mathcal{P}_{k-1}(E), \quad E \in \mathcal{E}_{h}(T), \\
& \text { (ii) } \mathbf{q} \mapsto \int_{F}\langle\mathbf{q} \times \mathbf{n}, \mathbf{p}\rangle \mathrm{d} \sigma, \quad \mathbf{p} \in \mathcal{P}_{k-2}(F)^{2}, \quad F \in \mathcal{F}_{h}(T) \text {, } \\
& \text { (ii) } \mathbf{q} \mapsto \int_{T}\langle\mathbf{q}, \mathbf{p}\rangle \mathrm{d} \mathbf{x}, \quad \mathbf{p} \in \mathcal{P}_{k-3}(T)^{3} \text {. }
\end{aligned}
$$

Here, polynomial spaces with negative degree are supposed to be empty. This specification of the degrees of freedom ensures that the global finite element space $\mathcal{N} \mathcal{D}_{k}\left(\Omega ; \mathcal{T}_{h}\right)$ is contained in $\boldsymbol{H}(\operatorname{curl} ; \Omega)$. Then, setting

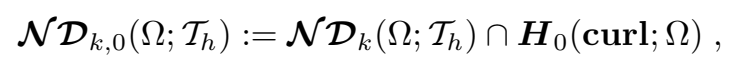

the curl-conforming finite element approximation of (3) is as follows: Find $\mathbf{u}_{h} \in \mathcal{N D}_{k, 0}\left(\Omega ; \mathcal{T}_{h}\right)$ such that

$$
\left(\chi \operatorname{curl} \mathbf{u}_{h}, \operatorname{curl} \mathbf{q}_{h}\right)_{\boldsymbol{L}^{2}(\Omega)}+\left(\beta \mathbf{u}_{h}, \mathbf{q}_{h}\right)_{\boldsymbol{L}^{2}(\Omega)}=\left(\mathbf{f}, \mathbf{q}_{h}\right)_{\boldsymbol{L}^{2}(\Omega)} \quad \forall \mathbf{q}_{h} \in \mathcal{N} \mathcal{D}_{k, 0}\left(\Omega ; \mathcal{T}_{h}\right) .
$$

We recall that Nédélec's finite elements provide affine equivalent families of finite elements in the sense of [27], if the vectorfields are subjected to a covariant transformation: For any $T \in \mathcal{T}_{h}$ write $\Phi: \widehat{T} \mapsto T$ for the affine mapping of a fixed reference tetrahedron $\widehat{T}$ to $T$ and define

$$
\mathfrak{F}(\mathbf{v})(\widehat{\mathbf{x}}):=D \Phi^{T}(\widehat{\mathbf{x}}) \mathbf{v}(\Phi(\widehat{\mathbf{x}})), \quad \widehat{\mathbf{x}} \in \widehat{T} .
$$


Then it turns out that $\mathcal{N} \mathcal{D}_{k}(\widehat{T})=\mathfrak{F}\left(\mathcal{N D}_{k}(T)\right)$ and the degrees of freedom are invariant under the transformation (7).

Edge elements provide one specimen of discrete differential forms [20,40]. This accounts for the exceptional property that the result that every curl-free vectorfield on a contractible domain is a gradient is preserved in purely discrete context. The role of the potentials is played by Lagrangian finite element functions

$$
S_{k, 0}\left(\Omega ; \mathcal{T}_{h}\right):=\left\{\phi_{h} \in C^{0}(\Omega),\left.\phi_{h}\right|_{\partial \Omega}=0,\left.\phi_{h}\right|_{T} \in \mathcal{P}_{k}(T)\right\}
$$

The following lemma is a special case of Theorem 20 of [40].

Lemma 1 (Discrete potentials). If the boundary $\partial \Omega$ is connected, then for any $\mathbf{q}_{h} \in \mathcal{N} \mathcal{D}_{k, 0}\left(\Omega ; \mathcal{T}_{h}\right), k \geq 1$, with curl $\mathbf{q}_{h}=0$ there exists a unique $\phi_{h} \in S_{k, 0}\left(\Omega ; \mathcal{T}_{h}\right)$ such that $\mathbf{q}_{h}=\operatorname{grad} \phi_{h}$.

\section{Residual BASED ERROR estimator}

We assume that $\tilde{\mathbf{u}}_{h} \in \mathcal{N}_{\mathcal{D}_{k, 0}}\left(\Omega ; \mathcal{T}_{h}\right)$ is some iterative approximation of the unique solution $\mathbf{u}_{h}$ of the curlconforming finite element solution of (6). It can be obtained, for instance, by the multigrid iterative solution process as developed in [39]. Denoting the total error by $\mathbf{e}:=\mathbf{u}-\tilde{\mathbf{u}}_{h}$, it is easy to see that e $\in \boldsymbol{H}_{0}(\mathbf{c u r l} ; \Omega)$ satisfies the defect equation

$$
(\chi \operatorname{curl} \mathbf{e}, \operatorname{curl} \mathbf{q})_{\boldsymbol{L}^{2}(\Omega)}+(\beta \mathbf{e}, \mathbf{q})_{\boldsymbol{L}^{2}(\Omega)}=r(\mathbf{q}) \quad \forall \mathbf{q} \in \boldsymbol{H}_{0}(\operatorname{curl} ; \Omega)
$$

where $r(\cdot)$ stands for the residual

$$
r(\mathbf{q}):=(\mathbf{f}, \mathbf{q})_{L^{2}(\Omega)}-\left(\chi \operatorname{curl} \tilde{\mathbf{u}}_{h}, \operatorname{curl} \mathbf{q}\right)_{L^{2}(\Omega)}-\left(\beta \tilde{\mathbf{u}}_{h}, \mathbf{q}\right)_{L^{2}(\Omega)}, \quad \mathbf{q} \in \boldsymbol{H}_{0}(\operatorname{curl} ; \Omega)
$$

The construction of the error estimator will be based on a direct splitting of the function space $\boldsymbol{H}_{0}(\mathbf{c u r l} ; \Omega)$

$$
\boldsymbol{H}_{0}(\operatorname{curl} ; \Omega)=\boldsymbol{H}_{0}^{0}(\operatorname{curl} ; \Omega) \oplus \boldsymbol{H}_{0}^{\perp}(\operatorname{curl} ; \Omega) \text {. }
$$

It may be labelled a " $\beta$-orthogonal" Helmholtz type decomposition, since we require

- Both $\boldsymbol{H}_{0}^{0}(\mathbf{c u r l} ; \Omega)$ and $\boldsymbol{H}_{0}^{\perp}(\mathbf{c u r l} ; \Omega)$ are closed subspaces of $\boldsymbol{H}_{0}(\mathbf{c u r l} ; \Omega)$.

- $\boldsymbol{H}_{0}^{0}(\operatorname{curl} ; \Omega):=\left\{\mathbf{q} \in \boldsymbol{H}_{0}(\operatorname{curl} ; \Omega) ; \operatorname{curl} \mathbf{q}=0\right\}$ is the kernel of the curl operator.

- $\left(\beta \mathbf{q}^{\perp}, \mathbf{q}^{0}\right)_{\boldsymbol{L}^{2}(\Omega)}=0$ for all $\mathbf{q}^{0} \in \boldsymbol{H}_{0}^{0}(\operatorname{curl} ; \Omega), \mathbf{q}^{\perp} \in \boldsymbol{H}_{0}^{\perp}(\operatorname{curl} ; \Omega)$.

Evidently, a decomposition complying with these requirement is also orthogonal with respect to the energy seminorm.

The following procedure furnishes a splitting of $\mathbf{q} \in \boldsymbol{H}_{0}(\mathbf{c u r l} ; \Omega)$ according to (10): First decompose $\mathbf{q}_{\Omega_{C}}=$ $\mathbf{u}^{0} \oplus \widetilde{\mathbf{u}}^{\perp}$, where $\mathbf{u}^{0}, \widetilde{\mathbf{u}}^{\perp} \in \boldsymbol{H}\left(\mathbf{c u r l} ; \Omega_{C}\right)$ and $\left(\beta \mathbf{u}^{0}, \widetilde{\mathbf{u}}^{\perp}\right)_{\boldsymbol{L}^{2}\left(\Omega_{C}\right)}=0$, curl $\mathbf{u}^{0}=0$. If $\operatorname{meas}\left(\partial \Omega \cap \partial \Omega_{C}\right)>0$, we also require that $\mathbf{u}^{0}, \widetilde{\mathbf{u}}^{\perp}$ have vanishing trace on $\partial \Omega$. Write $\mathbf{u}^{\perp}$ for the $\boldsymbol{H}(\mathbf{c u r l} ; \Omega)$-extension $\left(c f\right.$. [4]) of $\widetilde{\mathbf{u}}^{\perp}$ to $\Omega$. Then, let $\mathbf{v}^{\perp}$ be the unique vectorfield in $\boldsymbol{H}_{0}\left(\operatorname{curl} ; \Omega / \Omega_{C}\right)$ such that $\operatorname{curl}^{\perp}=\mathbf{c u r l} \mathbf{q}-\mathbf{c u r l} \mathbf{u}^{\perp}$ and $\mathbf{v}^{\perp} \perp \operatorname{Ker}(\mathbf{c u r l})$ in $\boldsymbol{H}_{0}\left(\mathbf{c u r l} ; \Omega / \Omega_{C}\right)$. Finally set

$$
\mathbf{q}^{\perp}:= \begin{cases}\widetilde{\mathbf{u}}^{\perp} & \text { in } \Omega_{C} \\ \mathbf{v}^{\perp}+\mathbf{u}^{\perp} & \text { in } \Omega / \Omega_{C} .\end{cases}
$$

The functions $\mathbf{q}^{\perp}$ thus constructed form a closed subspace of $\boldsymbol{H}_{0}(\mathbf{c u r l} ; \Omega)$. This can be seen by completeness arguments. 
Unfortunately, mere existence of such a decomposition is not enough; our theoretical examinations hinge on the following assumption:

Assumption 2. We assume that a splitting (10) with the above features can be found such that $\boldsymbol{H}_{0}^{\perp}(\mathbf{c u r l} ; \Omega)$ is continuously embedded in $\boldsymbol{H}^{1}(\Omega) \cap \boldsymbol{H}_{0}(\operatorname{curl} ; \Omega)$ and, moreover,

$$
\left|\mathbf{q}^{\perp}\right|_{H^{1}(\Omega)} \leq C(\Omega)\left\|\operatorname{curl} \mathbf{q}^{\perp}\right\|_{L^{2}(\Omega)} \quad \forall \mathbf{q}^{\perp} \in \boldsymbol{H}_{0}^{\perp}(\operatorname{curl} ; \Omega)
$$

Thus far, the statement of this assumption can be shown only if $\Omega_{C}=\Omega$ and $\beta, \chi$ are continuously differentiable and uniformly bounded away from zero $[6,37]$. In this case we simply use the true Helmholtzdecomposition.

A characterization of the kernel of the curl-operator is provided by the continuous version of Lemma 1 (cf. [37]):

Lemma 3. For any $\mathbf{q} \in \boldsymbol{H}_{0}^{0}(\mathbf{c u r l} ; \Omega)$ there exists a unique $\phi \in H_{0}^{1}(\Omega)$ such that $\mathbf{q}=\operatorname{grad} \phi$, provided that the boundary $\Gamma$ of $\Omega$ is connected.

If $\Gamma$ is not connected, i.e. $\Omega$ has embedded cavities, the entire kernel of curl is not provided by $\operatorname{grad} H_{0}^{1}(\Omega)$. This is only true modulo a space of small dimension (see Prop. 3.12 in [6]). To avoid technical difficulties we do not allow cavities in $\Omega$.

By means of the decomposition (10) we may split the total error according to $\mathbf{e}=\mathbf{e}^{0}+\mathbf{e}^{\perp}$, where $\mathbf{e}^{0} \in$ $\boldsymbol{H}_{0}^{0}(\operatorname{curl} ; \Omega)$ and $\mathbf{e}^{\perp} \in \boldsymbol{H}_{0}^{\perp}(\operatorname{curl} ; \Omega)$. We note that $\mathbf{e}^{0}$ represents the curl-free part of the total error whereas $\mathbf{e}^{\perp}$ stands for a " $\beta$-weakly solenoidal" part. As a matter of course, $\mathbf{e}^{0}$ is only meaningful in $\Omega_{C}$.

It readily follows from (8) that $\mathbf{e}^{0}$ and $\mathbf{e}^{\perp}$ are the unique solutions of the variational equations

$$
\begin{aligned}
\left(\beta \mathbf{e}^{0}, \mathbf{q}^{0}\right)_{L^{2}(\Omega)} & =r(\mathbf{q}) \quad \forall \mathbf{q}^{0} \in \boldsymbol{H}_{0}^{0}(\operatorname{curl} ; \Omega) \\
\left(\chi \operatorname{curl} \mathbf{e}^{\perp}, \operatorname{curl} \mathbf{q}^{\perp}\right)_{\boldsymbol{L}^{2}(\Omega)}+\left(\beta \mathbf{e}^{\perp}, \mathbf{q}^{\perp}\right)_{\boldsymbol{L}^{2}(\Omega)} & =r(\mathbf{q}) \quad \forall \mathbf{q}^{\perp} \in \boldsymbol{H}_{0}^{\perp}(\operatorname{curl} ; \Omega)
\end{aligned}
$$

The irrotational and the $\beta$-weakly solenoidal part of the error will be estimated separately. For simplicity, throughout the rest of this paper we assume the functions $\chi$ and $\beta$ to be elementwise constant.

As far as the irrotational part $\mathbf{e}^{0}$ is concerned, the estimate is based on the evaluation of the residual $r(\cdot)$ restricted to $\boldsymbol{H}_{0}^{0}(\mathbf{c u r l} ; \Omega)=\operatorname{grad} H_{0}^{1}(\Omega)$. In particular, exploiting that $\mathbf{f}$ is solenoidal, Green's formula yields

$$
\begin{aligned}
r(\operatorname{grad} v) & =\sum_{T \in \mathcal{T}_{h}}\left(\mathbf{f}-\beta \tilde{\mathbf{u}}_{h}, \operatorname{grad} v\right)_{\boldsymbol{L}^{2}(T)} \\
& =\sum_{T \in \mathcal{T}_{h}^{C}}\left(\operatorname{div} \beta \tilde{\mathbf{u}}_{h}, v\right)_{\boldsymbol{L}^{2}(T)}-\sum_{F \in \mathcal{F}_{h}^{C}}\left(\left[\left\langle\mathbf{n}, \beta \tilde{\mathbf{u}}_{h}\right\rangle\right]_{J}, v\right)_{L^{2}(F)},
\end{aligned}
$$

where $\left[\left\langle\mathbf{n}, \beta \tilde{\mathbf{u}}_{h}\right\rangle\right]_{J}$ denotes the jump of the normal component of the vector field $\beta \tilde{\mathbf{u}}_{h}$ across the interelement face $F \in \mathcal{F}_{h}^{\text {int }}$. It is defined as follows: If $F \in \mathcal{F}_{h}^{\text {int }}$ is the common face of two adjacent elements $T_{\text {in }}, T_{\text {out }} \in \mathcal{T}_{h}$ and $\mathbf{n}$ denotes the unit normal vector on $F$ directed towards the interior of $T_{\mathrm{in}}$, then

$$
\left[\langle\mathbf{n}, \mathbf{q}\rangle_{J}:=\langle\mathbf{n}, \mathbf{q}\rangle_{\mid F \subset T_{\text {out }}}-\langle\mathbf{n}, \mathbf{q}\rangle_{\mid F \subset T_{\text {in }}}\right.
$$

Note that $[\langle\cdot, \mathbf{n}\rangle]_{J}$ does not depend on the specification of $T_{\text {in }}$ and $T_{\text {out }}$.

As will be shown in Section 4, the upper and lower bounds for $\left\|\mathbf{e}^{0}\right\|_{\boldsymbol{L}^{2}(\Omega)}$ involve the error terms

$$
\eta^{(0)}:=\left(\sum_{T \in \mathcal{T}_{h}^{C}}\left(\eta_{0}^{T}\right)^{2}\right)^{1 / 2}+\left(\sum_{F \in \mathcal{F}_{h}^{C}}\left(\eta_{0}^{F}\right)^{2}\right)^{1 / 2}
$$


whose local contributions $\eta_{0}^{T}, \eta_{0}^{F}$ are given by

$$
\begin{array}{rlrl}
\eta_{0}^{T}:=h_{T}\left\|\operatorname{div} \sqrt{\beta} \tilde{\mathbf{u}}_{h}\right\|_{L^{2}(T)}, & & T \in \mathcal{T}_{h}^{C}, \\
\eta_{0}^{F}:=h_{F}^{1 / 2}\left\|\frac{1}{\sqrt{\beta_{A}}}\left[\left\langle\mathbf{n}, \beta \tilde{\mathbf{u}}_{h}\right\rangle\right]_{J}\right\|_{L^{2}(F)}, & F \in \mathcal{F}_{h}^{C},
\end{array}
$$

where $\beta_{A}$ is defined as the average on $F, \beta_{A}:=0.5\left(\beta_{\left.\right|_{T_{\text {out }}}}+\beta_{\left.\right|_{T_{\mathrm{in}}}}\right)$. The scaling in the different terms of the error estimator corresponds to the fact that we measure the error in the energy norm. For elements and faces outside $\bar{\Omega}_{C}$, we formally set the contributions $\eta_{0}^{T}$ and $\eta_{0}^{F}$ to zero.

The upper bound also involves the iteration error

$$
\eta_{\mathrm{it}}^{(0)}:=\left\|\sqrt{\beta}\left(\mathbf{u}_{h}-\tilde{\mathbf{u}}_{h}\right)\right\|_{L^{2}(\Omega)} .
$$

On the other hand, concerning bounds for $\mathbf{e}^{\perp}$, for $\mathbf{q} \in \boldsymbol{H}_{0}^{\perp}(\mathbf{c u r l} ; \Omega)$ the residual $r(\mathbf{q})$ can be written as

$$
\begin{aligned}
r(\mathbf{q}) & =\sum_{T \in \mathcal{T}_{h}}\left\{\left(\mathbf{f}-\beta \tilde{\mathbf{u}}_{h}, \mathbf{q}\right)_{\boldsymbol{L}^{2}(T)}-\left(\chi \operatorname{curl} \tilde{\mathbf{u}}_{h}, \operatorname{curl} \mathbf{q}\right)_{\boldsymbol{L}^{2}(T)}\right\} \\
& =\sum_{T \in \mathcal{T}_{h}}\left(\mathbf{f}-\operatorname{curl} \chi \operatorname{curl} \tilde{\mathbf{u}}_{h}-\beta \tilde{\mathbf{u}}_{h}, \mathbf{q}\right)_{\boldsymbol{L}^{2}(T)}-\sum_{F \in \mathcal{F}_{h}^{\operatorname{int}}}\left(\left[\mathbf{n} \times \chi \operatorname{curl} \tilde{\mathbf{u}}_{h}\right]_{J}, \mathbf{q}\right)_{\boldsymbol{L}^{2}(F)} .
\end{aligned}
$$

We note that a localization of the residual is not feasible due to the global character of the space $\boldsymbol{H}_{0}^{\perp}(\mathbf{c u r l} ; \Omega)$. Instead, we will use a localization by means of an interpolation with respect to the entire discrete space $\mathcal{N} \mathcal{D}_{k, 0}\left(\Omega ; \mathcal{T}_{h}\right)$. As we shall see in Section 5 , this is at the expense of a coupling between $\mathbf{e}^{0}$ and $\mathbf{e}^{\perp}$. However, this will not thwart the primary goal of obtaining an efficient and reliable estimate of the total error in the energy norm. In particular, the bounds for $\left\|\mathbf{e}^{\perp}\right\|_{\mathfrak{E} ; \Omega}$ comprise the error terms

$$
\begin{aligned}
\eta_{1}^{(1)} & :=\left(\sum_{T \in \mathcal{T}_{h}}\left(\eta_{1 ; 1}^{T}\right)^{2}\right)^{1 / 2}+\left(\sum_{F \in \mathcal{F}_{h}^{\text {int }}}\left(\eta_{1}^{F}\right)^{2}\right)^{1 / 2} \\
\eta_{2} & :=\left(\sum_{T \in \mathcal{T}_{h}}\left(\eta_{1 ; 2}^{T}\right)^{2}\right)^{1 / 2},
\end{aligned}
$$

with the local contributions $\eta_{1 ; \nu}^{T}, 1 \leq \nu \leq 2$, and $\eta_{1}^{F}$ given by

$$
\begin{aligned}
\eta_{1 ; 1}^{T}:=h_{T}\left\|\frac{1}{\sqrt{\chi}}\left(\pi_{h} \mathbf{f}-\mathbf{c u r l} \chi \mathbf{c u r l} \tilde{\mathbf{u}}_{h}-\beta \tilde{\mathbf{u}}_{h}\right)\right\|_{\boldsymbol{L}^{2}(T)}, & T \in \mathcal{T}_{h}, \\
\eta_{1 ; 2}^{T}:=h_{T}\left\|\frac{1}{\sqrt{\chi}}\left(\mathbf{f}-\pi_{h} \mathbf{f}\right)\right\|_{\boldsymbol{L}^{2}(T)}, & T \in \mathcal{T}_{h}, \\
\eta_{1}^{F}:=h_{F}^{1 / 2}\left\|\frac{1}{\sqrt{\chi A}}\left[\mathbf{n} \times \chi \operatorname{curl} \tilde{\mathbf{u}}_{h}\right]_{J}\right\|_{\boldsymbol{L}^{2}(F)}, & F \in \mathcal{F}_{h}^{\text {int }},
\end{aligned}
$$

where $\chi_{A}$ is defined as the average on $F, \chi_{A}:=0.5\left(\chi_{\left.\right|_{T_{\text {out }}}}+\chi_{\left.\right|_{T_{\mathrm{in}}}}\right)$. Here, $\pi_{h} \mathbf{f}$ denotes the $L^{2}$-projection of $\mathbf{f}$ onto $\prod_{T \in \mathcal{T}_{h}} \mathcal{P}_{k}(T)^{3}$.

Again, the iteration error

$$
\eta_{\mathrm{it}}^{(1)}:=\left\|\mathbf{u}_{h}-\tilde{\mathbf{u}}_{h}\right\|_{\mathfrak{E} ; \Omega}
$$


will enter the upper bound. Be aware that using a fast asymptotically optimal iteration scheme, we can quickly tell the truncation error from the size of the correction to the current iterate. Good bounds for $\eta_{\mathrm{it}}^{(0)}$ and $\eta_{\mathrm{it}}^{(1)}$ are at our disposal, thus.

As the main result of this paper we state the following a posteriori estimate for the total error e measured in the energy norm.

Theorem 4. Let $\eta_{1}:=\eta^{(0)}+\eta_{1}^{(1)}, \eta_{\mathrm{it}}:=\eta_{\mathrm{it}}^{(1)}+\eta_{\mathrm{it}}^{(0)}$ with $\eta^{(0)}, \eta_{1}^{(1)}, \eta_{\mathrm{it}}^{(1)}$, $\eta_{\mathrm{it}}^{(0)}$ given by (13), (12), (14), and (16), respectively. If assumption 2 holds true, then there exist constants $\gamma_{\nu}, \Gamma_{\nu}>0,1 \leq \nu \leq 2$, depending only on $\Omega, \underline{\chi}, \underline{\beta}, \bar{\chi}, \bar{\beta}$ and on the local geometry of $\mathcal{T}_{h_{0}}$ such that

$$
\gamma_{1} \eta_{1}-\gamma_{2} \eta_{2} \leq\|\mathbf{e}\|_{\mathfrak{E} ; \Omega} \leq \Gamma_{1}\left(\eta_{1}+\eta_{2}\right)+\Gamma_{2} \eta_{\mathrm{it}}
$$

Eventually, we need an estimate for the energy of the error on each element. Such an element oriented error estimator can be constructed by assigning half of the contribution of a face to either adjacent element. To offset the impact of jumps in the coefficients it is advisable to resort to additional scaling. As the actual error estimator we then get for each $T \in \mathcal{T}_{h}$

$$
\widehat{\eta}_{T}^{2}:=\left(\eta_{0}^{T}\right)^{2}+\left(\eta_{1 ; 1}^{T}\right)^{2}+\sum_{F \in \mathcal{F}(T) \cap \mathcal{F}_{h}^{\text {int }}} \frac{\beta_{\left.\right|_{T}}}{2 \beta_{A}}\left(\eta_{0}^{F}\right)^{2}+\frac{\chi_{\left.\right|_{T}}}{2 \chi_{A}}\left(\eta_{1}^{F}\right)^{2}
$$

Here $\beta_{A}$ and $\chi_{A}$ stand for the averages of the material parameters $\beta$ and $\chi$ over the two elements adjacent to the face $F$.

Since only local information is needed, the evaluation of $\widehat{\eta}_{T}$ is cheap. Low order numerical quadrature is sufficient to compute the local norms. Of course $\eta_{1 ; 2}^{T}$ is elusive, but $\pi_{h}$ has been chosen such that for smooth $\mathbf{f}$ this quantity can be expected to decrease faster than the other contributions to the error estimator.

For lowest order edge elements and locally constant coefficients, we can capitalize on the simple local ansatz space (5). First note that it contains only piecewise linear, divergence-free vectorfields. Therefore, $\chi \mathbf{c u r l} \tilde{\mathbf{u}}_{h}$ is locally constant and we end up with the simplified local error estimator

$$
\hat{\eta}_{T}^{2}=\frac{h_{T}^{2}}{\chi_{\left.\right|_{T}}}\left\|\pi_{h} \mathbf{f}-\beta \tilde{\mathbf{u}}_{h}\right\|_{\boldsymbol{L}^{2}(T)}^{2}+\sum_{F \in \mathcal{F}_{h}(T) \cap \mathcal{F}_{h}^{\mathrm{int}}}^{2} h_{F}\left(\frac{\beta_{\left.\right|_{T}}}{\beta_{A}}\left\|\left[\left\langle\mathbf{n}, \beta \tilde{\mathbf{u}}_{h}\right\rangle\right]_{J}\right\|_{L^{2}(F)}^{2}+\frac{\chi_{\left.\right|_{T}}}{\chi_{A}}\left\|\left[\mathbf{n} \times \chi \mathbf{c u r l} \tilde{\mathbf{u}}_{h}\right]_{J}\right\|_{L^{2}(F)}^{2}\right)
$$

Here, $\pi_{h}$ can be a suitable interpolation onto the space of piecewise linear vectorfields. Moreover, Gaussian quadrature formulas that are exact for quadratic polynomials on $T$ and $F$, respectively, can be used to evaluate all the norms. Thus, only the values of degrees of freedom in a neighborhood of $T$ and the local geometry of the mesh will show up in an explicit expression for $\hat{\eta}_{T}^{2}$.

\section{Estimator of the irRotational PART OF THE ERror}

Here, we will consider the irrotational part $\mathbf{e}^{0}$ of the error $\mathbf{e}$. Upper and lower bounds for $\left(\beta \mathbf{e}^{0}, \mathbf{e}^{0}\right)_{\boldsymbol{L}^{2}(\Omega)}$ will be established by means of $\eta^{(0)}$ and the iteration error. The starting point for the error analysis is the variational problem (11). The defect problem (8) restricted to the curl-free subspace of $\boldsymbol{H}_{0}(\mathbf{c u r l} ; \Omega)$ gives rise to the following uniformly positive definite variational problem on $H_{\Gamma}^{1}\left(\Omega_{C}\right)$ :

Find $\psi \in H_{\Gamma}^{1}\left(\Omega_{C}\right)$ such that

$$
(\beta \operatorname{grad} \psi, \operatorname{grad} \phi)_{L^{2}\left(\Omega_{C}\right)}=r(\operatorname{grad} \phi)=: \tilde{r}(\phi) \quad \forall \phi \in H_{\Gamma}^{1}\left(\Omega_{C}\right)
$$


Here, the space $H_{\Gamma}^{1}\left(\Omega_{C}\right)$ is defined as follows:

$$
H_{\Gamma}^{1}\left(\Omega_{C}\right):= \begin{cases}\left\{v \in H^{1}\left(\Omega_{C}\right) \mid(1, v)_{L^{2}\left(\Omega_{C}\right)}=0\right\}, & \text { if meas }\left(\partial \Omega_{C} \cap \partial \Omega\right)=0 \\ \left\{v \in H^{1}\left(\Omega_{C}\right) \mid v_{\mid \partial \Omega_{C} \cap \partial \Omega}=0\right\}, & \text { if meas }\left(\partial \Omega_{C} \cap \partial \Omega\right) \neq 0,\end{cases}
$$

and the residual $r(\cdot)$ is defined in (9). Thanks to Lemma 3 we have $\mathbf{e}^{0}=\operatorname{grad} \psi$ in $\Omega_{C}$. We will also write $\psi$ for the harmonic extension to a function in $H_{0}^{1}(\Omega)$.

Following the same lines as in the $H^{1}(\Omega)$-elliptic setting [55], the dual norm of $\tilde{r}(\cdot)$ restricted on $H_{\Gamma}^{1}\left(\Omega_{C}\right)$ provides bounds for $\left(\beta \mathbf{e}^{0}, \mathbf{e}^{0}\right)_{\boldsymbol{L}^{2}(\Omega)}$. The upper bound for $\left(\beta \mathbf{e}^{0}, \mathbf{e}^{0}\right)_{\boldsymbol{L}^{2}(\Omega)}$ is obtained by applying Green's formula. Observing Lemma 1 and Galerkin orthogonality

$$
\tilde{r}\left(\psi_{h}\right)=\left(\beta\left(\tilde{\mathbf{u}}_{h}-\mathbf{u}_{h}\right), \operatorname{grad} \psi_{h}\right)_{L^{2}\left(\Omega_{C}\right)}, \quad \psi_{h} \in S_{k}\left(\Omega_{C} ; \mathcal{T}_{h}\right)
$$

Here, $S_{k}\left(\Omega_{C} ; \mathcal{T}_{h}\right) \subset H_{\Gamma}^{1}\left(\Omega_{C}\right)$ denotes the $P_{k}$ conforming finite element space. We find for $\psi_{h} \in S_{k}\left(\Omega_{C} ; \mathcal{T}_{h}\right)$

$$
\left(\beta \mathbf{e}^{0}, \mathbf{e}^{0}\right)_{L^{2}(\Omega)}=\tilde{r}\left(\psi-\psi_{h}\right)+\left(\beta\left(\mathbf{u}_{h}-\tilde{\mathbf{u}}_{h}\right), \operatorname{grad} \psi_{h}\right)_{L^{2}\left(\Omega_{C}\right)} .
$$

In particular, $\tilde{r}\left(\psi_{h}\right)=0$ if the iteration error $\mathbf{u}_{h}-\tilde{\mathbf{u}}_{h}$ is zero. Upper bounds for the right side are obtained by a suitable choice of $\psi_{h}$. We set $\psi_{h}:=P_{h}^{k} \psi$, where $P_{h}^{k}: H_{\Gamma}^{1}\left(\Omega_{C}\right) \longrightarrow S_{k}\left(\Omega_{C} ; \mathcal{T}_{h}\right)$ is a locally defined projection-like operator satisfying approximation and stability properties

$$
\begin{aligned}
P_{h}^{k} \phi_{h} & =\phi_{h}, \quad \phi_{h} \in S_{k}\left(\Omega_{C} ; \mathcal{T}_{h}\right), \\
\left\|\phi-P_{h}^{k} \phi\right\|_{L^{2}(T)} & \leq C h_{T}\|\operatorname{grad} \phi\|_{L^{2}\left(D_{T}\right)}, \\
\left\|\phi-P_{h}^{k} \phi\right\|_{L^{2}(F)} & \leq C \sqrt{h_{F}}\|\operatorname{grad} \phi\|_{L^{2}\left(D_{F}\right)}, \\
\left\|\operatorname{grad} P_{h}^{k} \phi\right\|_{L^{2}(T)} & \leq C\|\operatorname{grad} \phi\|_{L^{2}\left(D_{T}\right)} .
\end{aligned}
$$

Here, $D_{T}$ and $D_{F}$ contain all elements in $\mathcal{T}_{h}$ sharing at least one vertex with $T$ and $F$, respectively. Such operators can be defined preserving boundary conditions by the use of dual basis functions. We refer to [51,53] for more details. In the case of a posteriori error estimates, the interpolation operator of Clément is very often used [30]. However, the Clément operator restricted on $S_{k}\left(\Omega_{C} ; \mathcal{T}_{h}\right)$ is not the identity. Using Green's formula, the approximation properties $(21),(22)$ of $P_{h}^{k}$, and $\operatorname{div} \mathbf{f}=0$, we get

$$
\begin{aligned}
\tilde{r}\left(\psi-P_{h}^{k} \psi\right)= & \sum_{T \in \mathcal{T}_{h}^{C}}\left(\operatorname{div} \beta \tilde{\mathbf{u}}_{h}, \psi-P_{h}^{k} \psi\right)_{L^{2}(T)}-\sum_{F \in \mathcal{F}_{h}^{C}}\left(\left[\left\langle\mathbf{n}, \beta \tilde{\mathbf{u}}_{h}\right\rangle\right]_{J}, \psi-P_{h}^{k} \psi\right)_{L^{2}(F)} \\
\leq & C\left(\sum_{T \in \mathcal{T}_{h}^{C}} h_{T}^{2}\left\|\operatorname{div} \sqrt{\beta} \tilde{\mathbf{u}}_{h}\right\|_{L^{2}(T)}^{2}\left\|\sqrt{\beta} \mathbf{e}^{0}\right\|_{\boldsymbol{L}^{2}\left(\Omega_{C}\right)}\right. \\
& +C\left(\sum_{F \in \mathcal{F}_{h}^{C}} \frac{h_{F}}{\sqrt{\beta_{A}}}\left\|\left[\left\langle\mathbf{n}, \beta \tilde{\mathbf{u}}_{h}\right\rangle\right]_{J}\right\|_{L^{2}(F)}^{2}\right)^{\frac{1}{2}}\left\|\sqrt{\beta} \mathbf{e}^{0}\right\|_{\boldsymbol{L}^{2}\left(\Omega_{C}\right)} \cdot
\end{aligned}
$$

Here, we set $\left.\left[\left\langle\mathbf{n}, \beta \tilde{\mathbf{u}}_{h}\right\rangle\right]_{J}\right|_{F}:=0$ if $F \subset \partial \Omega_{C} \cap \partial \Omega$ and $\left.\left[\left\langle\mathbf{n}, \beta \tilde{\mathbf{u}}_{h}\right\rangle\right]_{J}\right|_{F}:=\beta \tilde{\mathbf{u}}_{h} \mathbf{n}_{T}$ if $F \subset \partial \Omega_{C} \backslash \partial \Omega, F \subset \partial T$. An upper bound for the second term on the right side of (20) is obtained by means of (23). The stability of the projection-like operator $P_{h}^{k}$ yields

$$
\left(\beta\left(\tilde{\mathbf{u}}_{h}-\tilde{\mathbf{u}}_{h}\right), \operatorname{grad} P_{h}^{k} \psi\right)_{L^{2}\left(\Omega_{C}\right)} \leq C\left\|\sqrt{\beta}\left(\mathbf{u}_{h}-\tilde{\mathbf{u}}_{h}\right)\right\|_{L^{2}\left(\Omega_{C}\right)}\left\|\sqrt{\beta} \mathbf{e}^{0}\right\|_{L^{2}\left(\Omega_{C}\right)}
$$


and thus

$$
\left\|\sqrt{\beta} \mathbf{e}^{0}\right\|_{L^{2}\left(\Omega_{C}\right)} \leq C\left(\eta^{(0)}+\eta_{\mathrm{it}}^{(0)}\right) .
$$

We point out that in the proof for the upper bound of $\left\|\sqrt{\beta} \mathbf{e}^{0}\right\|_{L^{2}\left(\Omega_{C}\right)}$, the stability and approximation properties of a suitable quasi-interpolation operator from $H_{\Gamma}^{1}\left(\Omega_{C}\right)$ onto $S_{k}\left(\Omega_{C} ; \mathcal{T}_{h}\right)$ play an important role.

We establish the lower bound exploiting properties of local bubble functions: Two different types of bubble functions $\lambda_{T}$ and $\lambda_{F}$ defined on $T$ are used. We set

$$
\lambda_{T}:=256 \prod_{l=1}^{4} \lambda_{l ; T}, \quad \lambda_{F}:=27 \prod_{l=1}^{3} \lambda_{F_{l} ; T},
$$

where $\lambda_{l ; T}, 1 \leq l \leq 4$ are the barycentric coordinates of $T$ associated with the vertices $p_{l}$. The face $F$ is spanned by the vertices $p_{F_{l}}, 1 \leq l \leq 3$ of $T$. We set out from the following norm equivalences

$$
\begin{array}{ll}
\left\|\lambda_{T}^{1 / 2} \phi_{h}\right\|_{L^{2}(T)} \leq\left\|\phi_{h}\right\|_{L^{2}(T)} \leq C\left\|\lambda_{T}^{1 / 2} \phi_{h}\right\|_{L^{2}(T)} & \forall \phi_{h} \in P_{k}(T) \\
\left\|\lambda_{F}^{1 / 2} \phi_{h}\right\|_{L^{2}(F)} \leq\left\|\phi_{h}\right\|_{L^{2}(F)} \leq C\left\|\lambda_{F}^{1 / 2} \phi_{h}\right\|_{L^{2}(F)} & \forall \phi_{h} \in P_{k}(F) .
\end{array}
$$

The independence of the constants of the elements and faces can be seen by an affine equivalence argument. The two local components of the error estimator are estimated separately. In a first step, we consider the element oriented contribution and show local upper bounds for $\eta_{0}^{T}$. Using (24), an inverse inequality and taking into account that $\lambda_{T}=0$ on the boundary of $T$, we find

$$
\begin{aligned}
\frac{\left(\eta_{0}^{T}\right)^{2}}{h_{T}^{2}} & =\left\|\operatorname{div} \sqrt{\beta} \tilde{\mathbf{u}}_{h}\right\|_{L^{2}(T)}^{2} \leq C \int_{T} \frac{1}{\beta}\left(\operatorname{div} \beta \tilde{\mathbf{u}}_{h}\right)^{2} \lambda_{T} \mathrm{~d} \mathbf{x} \\
& \leq-C \int_{T} \beta\left\langle\tilde{\mathbf{u}}_{h}, \operatorname{grad}\left(\lambda_{T} \operatorname{div} \tilde{\mathbf{u}}_{h}\right)\right\rangle \mathrm{d} \mathbf{x}=C r\left(\lambda_{T} \operatorname{div} \tilde{\mathbf{u}}_{h}\right) \\
& \leq-C\left(\beta \mathbf{e}^{0}, \operatorname{grad}\left(\lambda_{T} \operatorname{div} \tilde{\mathbf{u}}_{h}\right)\right)_{\boldsymbol{L}^{2}(T)} \leq C\left\|\sqrt{\beta} \mathbf{e}^{0}\right\|_{L^{2}(T)}\left\|\operatorname{grad}\left(\lambda_{T} \operatorname{div} \sqrt{\beta} \tilde{\mathbf{u}}_{h}\right)\right\|_{L^{2}(T)} \\
& \leq \frac{C}{h_{T}}\left\|\sqrt{\beta} \mathbf{e}^{0}\right\|_{L^{2}(T)}\left\|\operatorname{div} \sqrt{\beta} \tilde{\mathbf{u}}_{h}\right\|_{L^{2}(T)} .
\end{aligned}
$$

Local upper bounds for $\eta_{0}^{F}$ are obtained in a similar fashion. The basic tools to establish the bounds are (24), Green's formula and an inverse estimate. Let $T_{1}$ and $T_{2}$ be the elements such that $\partial T_{1} \cap \partial T_{2}=F$. Then we can transform the $L^{2}$-norm on $F$ into an integral on $T_{1}$ and $T_{2}$ :

$$
\begin{aligned}
\frac{\left(\eta_{0}^{F}\right)^{2}}{h_{F}} & =\left\|\frac{1}{\sqrt{\beta_{A}}}\left[\left\langle\mathbf{n}, \beta \tilde{\mathbf{u}}_{h}\right\rangle\right]_{J}\right\|_{L^{2}(F)}^{2} \leq C \int_{F} \frac{1}{\beta_{A}}\left[\left\langle\mathbf{n}, \beta \tilde{\mathbf{u}}_{h}\right\rangle\right]_{J}^{2} \lambda_{F} \mathrm{~d} \sigma \\
& \leq \frac{C}{\beta_{A}} \sum_{i=1}^{2} \int_{T_{i}}\left(\beta\left\langle\tilde{\mathbf{u}}_{h}, \operatorname{grad}\left(\left[\left\langle\mathbf{n}, \beta \tilde{\mathbf{u}}_{h}\right\rangle\right]_{J ; T_{i}} \lambda_{F}\right)\right\rangle+\operatorname{div} \beta \tilde{\mathbf{u}}_{h}\left[\left\langle\mathbf{n}, \beta \tilde{\mathbf{u}}_{h}\right\rangle\right]_{J ; T_{i}} \lambda_{F}\right) \mathrm{d} \mathbf{x} .
\end{aligned}
$$

Here, the function $\left[\left\langle\mathbf{n}, \beta \tilde{\mathbf{u}}_{h}\right\rangle\right]_{J}$ on $F$ is extended by a continuous piecewise polynomial function $\left[\left\langle\mathbf{n}, \beta \tilde{\mathbf{u}}_{h}\right\rangle\right]_{J ; T_{i}}$ to $T_{1} \cup T_{2}$ such that

$$
\left\|\left[\left\langle\mathbf{n}, \beta \tilde{\mathbf{u}}_{h}\right\rangle\right]_{J ; T_{i}}\right\|_{L^{2}\left(T_{i}\right)} \leq C h_{T_{i}}^{1 / 2}\left\|\left[\left\langle\mathbf{n}, \beta \tilde{\mathbf{u}}_{h}\right\rangle\right]_{J}\right\|_{L^{2}(F)} .
$$


Now, we are in a position to give an upper bound for $\eta_{0}^{F}$ by means of $\eta_{0}^{T}$ and $\mathbf{e}^{0}$

$$
\frac{\left(\eta_{0}^{F}\right)^{2}}{h_{F}} \leq C \sum_{i=1}^{2}\left(h_{T_{i}}^{-1 / 2}\left\|\sqrt{\beta} \mathbf{e}^{0}\right\|_{L^{2}\left(T_{i}\right)}+h_{T_{i}}^{1 / 2}\left\|\operatorname{div} \sqrt{\beta} \tilde{\mathbf{u}}_{h}\right\|_{L^{2}\left(T_{i}\right)}\right) \frac{\eta_{0}^{F}}{h_{F}^{1 / 2}}
$$

Using the upper bound for $\eta_{0}^{T}$, we finally obtain an upper bound for $\eta_{0}^{F}$ in terms of $\left\|\sqrt{\beta} \mathbf{e}^{0}\right\|_{L^{2}\left(T_{1} \cup T_{2}\right)}$, and thus

$$
\eta_{0}^{T}+\sum_{F \subset \partial T} \eta_{0}^{F} \leq C\left\|\sqrt{\beta} \mathbf{e}^{0}\right\|_{L^{2}\left(\Omega_{T}\right)}
$$

where $\Omega_{T}$ is the union of all elements $T^{\prime}$ sharing at least one face with $T$. Keep in mind that $C>0$ does not depend on the meshsize. This is the desired local lower bound for the curl-free part of the error.

\section{Estimation OF THE $\beta$-WEAKLY SOLENOIDAL PART OF THE ERROR}

To establish upper bounds for the " $\beta$-weakly solenoidal" part $\mathbf{e}^{\perp}$ of the error, we basically follow the same ideas as before. Now, we have to apply Green's formula for the curl-operator. Furthermore, the scalar locally defined quasi-interpolation operator $P_{h}^{k}$ will be replaced by a vector-valued counterpart $\mathfrak{P}_{h}^{k}$. Again, nodal interpolation is not suitable, since the degrees of freedom located on edges cannot be extended to continuous functionals on $\boldsymbol{H}^{1}(\Omega)[6]$.

Lemma 5. For $T \in \mathcal{T}_{h}, F \in \mathcal{F}_{h}^{\text {int }}$ and $E \in \mathcal{E}_{h}^{\text {int }}$, let $D_{T}^{1}, D_{F}^{1}$, and $D_{E}$ be given by

$$
\begin{aligned}
D_{E} & :=\cup\left\{T \in \mathcal{T}_{h}, E \in \mathcal{E}_{h}(T)\right\}, \\
D_{T}^{1} & :=\cup\left\{D_{E}, E \in \mathcal{E}_{h}(T)\right\}, \\
D_{F}^{1} & :=\cup\left\{D_{E}, E \in \mathcal{E}_{h}(F)\right\} .
\end{aligned}
$$

There exists a linear projection $\mathfrak{P}_{h}^{k}: \boldsymbol{H}^{1}(\Omega) \cap \boldsymbol{H}_{0}(\mathbf{c u r l} ; \Omega) \mapsto \mathcal{N D}_{k, 0}\left(\Omega ; \mathcal{T}_{h}\right)$ such that for all $\mathbf{q} \in \boldsymbol{H}^{1}(\Omega)$

$$
\begin{aligned}
\left\|\mathfrak{P}_{h}^{k} \mathbf{q}\right\|_{L^{2}(T)} & \leq d_{1}\|\mathbf{q}\|_{\boldsymbol{H}^{1}\left(D_{T}^{1}\right)}, \\
\left\|\mathbf{c u r l} \mathfrak{P}_{h}^{k} \mathbf{q}\right\|_{L^{2}(T)} & \leq d_{2}|\mathbf{q}|_{\boldsymbol{H}^{1}\left(D_{F}^{1}\right)}, \\
\left\|\mathbf{q}-\mathfrak{P}_{h}^{k} \mathbf{q}\right\|_{L^{2}(T)} & \leq d_{3} h_{T}|\mathbf{q}|_{\boldsymbol{H}^{1}\left(D_{T}^{1}\right)}, \\
\left\|\mathbf{q}-\mathfrak{P}_{h}^{k} \mathbf{q}\right\|_{L^{2}(F)} & \leq d_{4} \sqrt{h_{F}}|\mathbf{q}|_{\boldsymbol{H}^{1}\left(D_{F}^{1}\right)}
\end{aligned}
$$

where the constants $d_{1}, d_{2}, d_{3}, d_{4}>0$ do neither depend on $\mathbf{q}$ nor on $T$, but only on the shape regularity of the mesh $\mathcal{T}_{h}$.

Proof. Details will only be given for the lowest order case $k=1$, where the degrees of freedom are plain path integrals along the edges of the elements. We adopt the notation $\mathbf{w}_{E}, E \in \mathcal{E}_{h}$, for the canonical basis function of $\mathcal{N} \mathcal{D}_{1}\left(\Omega ; \mathcal{T}_{h}\right)$ attached to edge $E$.

Pick any $F \in \mathcal{F}_{h}$ with vertices $\left\{\mathbf{a}_{1}, \mathbf{a}_{2}, \mathbf{a}_{3}\right\}$ and edges $\left\{E_{1}, E_{2}, E_{3}\right\}=\mathcal{E}_{h}(F)$. With $e_{i}, i=1,2,3$, we abbreviate the length of the edge $E_{i}$ and set $s^{2}:=e_{1}^{2}+e_{2}^{2}+e_{3}^{2}$. Then define $A:=\left(a_{i j}\right) \in \mathbb{R}^{3,3}$ by

$$
A=6\left(\begin{array}{lll}
3 / 4 s^{2}-e_{1}^{2} & 1 / 4 s^{2}-e_{3}^{2} & 1 / 4 s^{2}-e_{2}^{2} \\
1 / 4 s^{2}-e_{3}^{2} & 3 / 4 s^{2}-e_{2}^{2} & 1 / 4 s^{2}-e_{1}^{2} \\
1 / 4 s^{2}-e_{2}^{2} & 1 / 4 s^{2}-e_{1}^{2} & 3 / 4 s^{2}-e_{3}^{2}
\end{array}\right)^{-1}\left(\begin{array}{ccc}
e_{1}^{-1} & 0 & 0 \\
0 & e_{2}^{-1} & 0 \\
0 & 0 & e_{3}^{-1}
\end{array}\right)
$$


and set

$$
\phi_{j}^{F}(\mathbf{x}):= \pm \sum_{k=1}^{3} a_{k j}\left(\mathbf{x}-\mathbf{a}_{k}\right), \quad \mathbf{x} \in T .
$$

The sign takes into account the orientation of the edges, which will be irrelevant for our considerations. Straightforward computations show

$$
\int_{F}\left\langle\mathbf{w}_{E_{i}}(\cdot) \times \mathbf{n}, \phi_{j}^{F}(\cdot)\right\rangle \mathrm{d} \sigma=\delta_{i j}, \quad i, j=1,2,3 .
$$

Following this procedure, for each face $F$ we construct three functions $\phi_{E}^{F}, E \in \mathcal{E}_{h}(F)$, indexed by the edges of the face, so that they satisfy relations like (31).

Next, to each $E \in \mathcal{E}_{h}$ we assign one of its adjacent faces and call it $F_{E} \in \mathcal{F}_{h}$. We have to comply with the restriction that for $E \in \mathcal{E}_{h}^{\Gamma}$ also $F_{E} \in \mathcal{F}_{h}^{\Gamma}$. Then we can define

$$
\mathfrak{P}_{h}^{k}(\mathbf{q}):=\sum_{E \in \mathcal{E}_{F_{F}}} \int_{F_{E}}\left\langle\mathbf{q}(\cdot) \times \mathbf{n}, \boldsymbol{\phi}_{E}^{F}(\cdot)\right\rangle \mathrm{d} \sigma \cdot \mathbf{w}_{E} .
$$

By virtue of (31) this defines a projection. Obviously, boundary conditions are respected.

From the formulas (29) and (30) we conclude that

$$
\left\|\phi_{E}^{F}\right\|_{L^{\infty}(F)} \leq C h_{F}^{-2} \Rightarrow\left\|\phi_{E}^{F}\right\|_{L^{2}(F)} \leq C h_{F}^{-1} .
$$

Here $C>0$ represents a generic constant that depends on the angles of the face only. Thanks to shape regularity of $\mathcal{T}_{h}$, this means that the constants can be chosen independently of $F$. As all $\phi_{E}^{F}, E \in \mathcal{E}_{h}(F)$, are linear polynomials, the inverse estimate

$$
\left\|\phi_{E}^{F}\right\|_{\boldsymbol{H}^{-\frac{1}{2}(F)}} \leq C h_{F}^{-1 / 2}\left\|\phi_{E}^{F}\right\|_{\boldsymbol{L}^{2}(F)}, \quad E \in \mathcal{E}_{h}(F),
$$

follows from shape regularity. It yields for all $E \in \mathcal{E}_{h}(F), F \in \mathcal{F}_{h}$,

$$
\begin{aligned}
\int_{F}\left\langle\mathbf{q}(\cdot) \times \mathbf{n}, \phi_{E}^{F}(\cdot)\right\rangle \mathrm{d} \sigma & \leq\|\mathbf{q}\|_{\boldsymbol{H}^{\frac{1}{2}}(F)}\left\|\phi_{E}^{F}\right\|_{\boldsymbol{H}^{-\frac{1}{2}}(F)} \\
& \leq C h_{F}^{-1 / 2}\|\mathbf{q}\|_{\boldsymbol{H}^{\frac{1}{2}(F)}} \leq C h^{-1 / 2}\|\mathbf{q}\|_{H^{1}\left(D_{F}^{1}\right)} .
\end{aligned}
$$

Now, consider $T \in \mathcal{T}_{h}$ and recall that, again as a consequence of shape regularity, for $\mathbf{v}_{h} \in \mathcal{N} \mathcal{D}_{1}\left(\Omega ; \mathcal{T}_{h}\right)$ we have (cf. [39])

$$
\left\|\mathbf{v}_{h}\right\|_{L^{2}(T)}^{2} \leq C h_{T} \sum_{E \in \mathcal{E}_{h}(T)}\left|\int_{E}\left\langle\mathbf{v}_{h}, \mathbf{t}_{E}\right\rangle \mathrm{d} s\right|^{2}
$$


Combining this with estimate (33) and the definition (32) we end up with the estimate (25):

$$
\begin{aligned}
\left\|\mathfrak{P}_{h} \mathbf{q}\right\|_{L^{2}(T)}^{2} & \leq C h_{T} \sum_{E \in \mathcal{E}_{h}(T)}\left|\int_{F_{E}}\left\langle\mathbf{q}(\cdot) \times \mathbf{n}, \phi_{E}^{F}(\cdot)\right\rangle \mathrm{d} \sigma\right|^{2} \\
& \leq C h_{T} \sum_{E \in \mathcal{E}_{h}(T)} h_{F_{E}}^{-1}\|\mathbf{q}\|_{H^{1}\left(D_{F}^{1}\right)}^{2} \\
& \leq C\|\mathbf{q}\|_{H^{1}\left(D_{T}^{1}\right)}^{2} .
\end{aligned}
$$

The final estimate is possible, as shape regularity ensures that the number of elements sharing an edge is uniformly bounded.

To get the remaining estimates (26)-(28), we have to resort to affine equivalence techniques, mapping $T$ to a reference simplex, where a Bramble-Hilbert argument ( $c f .[27])$ is available. We will skip the technicalities and refer to [52] for an application of those tricks to edge elements.

When we compare the properties of $P_{h}^{k}$ and $\mathfrak{P}_{h}^{k}$, we see that both operators are stable and possess the same approximation properties. The variational problem (11) yields an expression for the energy norm of $\mathbf{e}^{\perp}$ :

$$
\left\|\mathbf{e}^{\perp}\right\|_{\mathfrak{E} ; \Omega}^{2}=r\left(\mathbf{e}^{\perp}\right)=r\left(\mathbf{e}^{\perp}-\mathfrak{P}_{h}^{k} \mathbf{e}^{\perp}\right)+r\left(\mathfrak{P}_{h}^{k} \mathbf{e}^{\perp}\right)
$$

We apply Green's formula on the first term on the right side and find

$$
\begin{aligned}
r\left(\mathbf{e}^{\perp}-\mathfrak{P}_{h}^{k} \mathbf{e}^{\perp}\right)= & \sum_{T \in \mathcal{T}_{h}}\left(\mathbf{f}-\beta \tilde{\mathbf{u}}_{h}-\operatorname{curl}\left(\chi \operatorname{curl} \tilde{\mathbf{u}}_{h}\right), \mathbf{e}^{\perp}-\mathfrak{P}_{h}^{k} \mathbf{e}^{\perp}\right)_{L^{2}(T)} \\
& -\sum_{F \in \mathcal{F}_{h}^{\text {int }}}\left(\left[\mathbf{n} \times \chi \operatorname{curl} \tilde{\mathbf{u}}_{h}\right]_{J}, \mathbf{e}^{\perp}-\mathfrak{P}_{h}^{k} \mathbf{e}^{\perp}\right)_{\boldsymbol{L}^{2}(F)} .
\end{aligned}
$$

By means of the approximation properties $(27),(28)$ of $\mathfrak{P}_{h}^{k}$, it is easy to obtain upper bounds for $r\left(\mathbf{e}^{\perp}-\mathfrak{P}_{h}^{k} \mathbf{e}^{\perp}\right)$

$$
\begin{aligned}
r\left(\mathbf{e}^{\perp}-\mathfrak{P}_{h}^{k} \mathbf{e}^{\perp}\right) \leq & C\left(\sum_{T \in \mathcal{T}_{h}}\left\|\frac{h_{T}}{\sqrt{\chi}}\left(\mathbf{f}-\beta \tilde{\mathbf{u}}_{h}-\operatorname{curl}\left(\chi \operatorname{curl} \tilde{\mathbf{u}}_{h}\right)\right)\right\|_{\mathbf{L}^{2}(T)}^{2}\right)^{1 / 2}\left|\sqrt{\chi} \mathbf{e}^{\perp}\right|_{\boldsymbol{H}^{1}(\Omega)} \\
& +C\left(\sum_{F \in \mathcal{F}_{h}^{\text {fint }}}\left\|\left[\mathbf{n} \times \chi \operatorname{curl} \tilde{\mathbf{u}}_{h}\right]_{J}\right\|_{\boldsymbol{L}^{2}(F)}^{2}\right)^{1 / 2}\left|\sqrt{\chi} \mathbf{e}^{\perp}\right|_{\boldsymbol{H}^{1}(\Omega)}
\end{aligned}
$$

Due to the Galerkin orthogonality, the second part $r\left(\mathfrak{P}_{h}^{k} \mathbf{e}^{\perp}\right)$ is equal to zero, if the iteration error is zero. The estimate for this term involves the stability $(25),(26)$ of the operator $\mathfrak{P}_{h}^{k}$

$$
\begin{aligned}
r\left(\mathfrak{P}_{h}^{k} \mathbf{e}^{\perp}\right) & =\left(\beta\left(\mathbf{u}_{h}-\tilde{\mathbf{u}}_{h}\right), \mathfrak{P}_{h}^{k} \mathbf{e}^{\perp}\right)_{L^{2}(\Omega)}+\left(\chi \operatorname{curl}\left(\mathbf{u}_{h}-\tilde{\mathbf{u}}_{h}\right), \operatorname{curl} \mathfrak{P}_{h}^{k} \mathbf{e}^{\perp}\right)_{L^{2}(\Omega)} \\
& \leq\left\|\mathbf{u}_{h}-\tilde{\mathbf{u}}_{h}\right\|_{\mathfrak{E} ; \Omega}\left\|\mathfrak{P}_{h}^{k} \mathbf{e}^{\perp}\right\|_{\mathfrak{E} ; \Omega} \leq C\left\|\mathbf{u}_{h}-\tilde{\mathbf{u}}_{h}\right\|_{\mathfrak{E} ; \Omega}\left\|\mathbf{e}^{\perp}\right\|_{\boldsymbol{H}^{1}(\Omega)} .
\end{aligned}
$$

At this point we have to resort to the regularity Assumption 2 to switch from $\boldsymbol{H}^{1}(\Omega)$-norms back to the relevant energy norm. Taking Assumption 2 for granted we can combine the upper estimates for $r\left(\mathbf{e}^{\perp}-\mathfrak{P}_{h}^{k} \mathbf{e}^{\perp}\right), r\left(\mathfrak{P}_{h}^{k} \mathbf{e}^{\perp}\right)$. Thus, an upper bound for $\left\|\mathbf{e}^{\perp}\right\|_{\mathfrak{E} ; \Omega}$ is provided by the sum of $\eta_{1}^{(1)}, \eta_{2}$ and $\eta_{\mathrm{it}}^{(1)}$.

To obtain a lower bound for $\left\|\mathbf{e}^{\perp}\right\|_{\mathfrak{E} ; \Omega}$, we consider the local contributions of $\eta_{1}^{(1)}$ separately. The global error is localized by means of the bubble functions $\lambda_{T}$ and $\lambda_{F}$ introduced in Section 4 . We start with an upper bound 
for $\eta_{1 ; 1}^{T}$ and set $\mathbf{j}_{h}:=\pi_{h} \mathbf{f}-\beta \tilde{\mathbf{u}}_{h}-\operatorname{curl}\left(\chi \operatorname{curl} \tilde{\mathbf{u}}_{h}\right)$

$$
\begin{aligned}
\frac{\chi_{\left.\right|_{T}}\left(\eta_{1 ; 1}^{T}\right)^{2}}{h_{T}^{2}} & =\left\|\pi_{h} \mathbf{f}-\beta \tilde{\mathbf{u}}_{h}-\operatorname{curl}\left(\chi \operatorname{curl} \tilde{\mathbf{u}}_{h}\right)\right\|_{L^{2}(T)}^{2} \\
& \leq C \int_{T}\left\langle\pi_{h} \mathbf{f}-\beta \tilde{\mathbf{u}}_{h}-\operatorname{curl}\left(\chi \operatorname{curl} \tilde{\mathbf{u}}_{h}\right), \mathbf{j}_{h} \lambda_{T}\right\rangle \mathrm{d} \mathbf{x} .
\end{aligned}
$$

Observing that $\left(\lambda_{T} \mathbf{j}_{h}\right)_{\left.\right|_{\partial T}}=0$ and applying Green's formula yields

$$
\begin{aligned}
\frac{\chi_{\left.\right|_{T}}\left(\eta_{1 ; 1}^{T}\right)^{2}}{h_{T}^{2}} & \leq C\left(r\left(\lambda_{T} \mathbf{j}_{h}\right)-\int_{T}\left\langle\mathbf{f}-\pi_{h} \mathbf{f}, \lambda_{T} \mathbf{j}_{h}\right\rangle \mathrm{d} \mathbf{x}\right) \\
& \leq C\left(\|\mathbf{e}\|_{\mathfrak{E} ; T}\left\|\lambda_{T} \mathbf{j}_{h}\right\|_{\mathfrak{E} ; T}+\left\|\mathbf{f}-\pi_{h} \mathbf{f}\right\|_{\boldsymbol{L}^{2}(T)}\left\|\lambda_{T} \mathbf{j}_{h}\right\|_{\boldsymbol{L}^{2}(T)}\right) \\
& \leq C\left(h_{T}^{-1}\|\mathbf{e}\|_{\mathfrak{E} ; T}+\frac{1}{\sqrt{\chi_{\left.\right|_{T}}}}\left\|\mathbf{f}-\pi_{h} \mathbf{f}\right\|_{\boldsymbol{L}^{2}(T)}\right)\left\|\sqrt{\chi} \mathbf{j}_{h}\right\|_{\boldsymbol{L}^{2}(T)},
\end{aligned}
$$

and we get

$$
\eta_{1 ; 1}^{T} \leq C\left(\|\mathbf{e}\|_{\mathfrak{E} ; T}+\eta_{1 ; 2}^{T}\right)
$$

We remark, that in general $\lambda_{T} \mathbf{j}_{h}$ is not an element of $\boldsymbol{H}_{0}^{\perp}(\mathbf{c u r l} ; \Omega)$. As a consequence, the upper bound for $\eta_{1 ; 1}^{T}$ involves not only the solenoidal part of the error $\mathbf{e}^{\perp}$ but the total error $\mathbf{e}$. The estimate for an upper bound for $\eta_{1}^{F}$ follows the same lines. Here, we use the bubble function $\lambda_{F}$. The face contribution $\eta_{1}^{F}$ involves the two adjacent elements $T_{1}$ and $T_{2}, \partial T_{1} \cap \partial T_{2}$. We extend the jump $\left[\mathbf{n} \times \chi \mathbf{c u r l} \tilde{\mathbf{u}}_{h}\right]_{J}$ defined on the face $F$ to a polynomial function defined on $T_{1}$ and $T_{2}$ such that for $1 \leq i \leq 2$

$$
\left\|\left[\mathbf{n} \times \chi \operatorname{curl} \tilde{\mathbf{u}}_{h}\right]_{J ; T_{i}}\right\|_{\boldsymbol{L}^{2}\left(T_{i}\right)} \leq C h_{T_{i}}^{1 / 2}\left\|\left[\mathbf{n} \times \chi \operatorname{curl} \tilde{\mathbf{u}}_{h}\right]_{J}\right\|_{L^{2}(F)} .
$$

Setting $\mathbf{j}_{\left.\right|_{T_{i}}}:=\left[\mathbf{n} \times \chi \operatorname{curl} \tilde{\mathbf{u}}_{h}\right]_{J ; T_{i}}$ and using the norm equivalence (24), we find

$$
\frac{\chi_{A}\left(\eta_{1}^{F}\right)^{2}}{h_{F}}=\left\|\left[\mathbf{n} \times \chi \operatorname{curl} \tilde{\mathbf{u}}_{h}\right]_{J}\right\|_{\boldsymbol{L}^{2}(F)}^{2} \leq C \int_{F}\left\langle\left[\mathbf{n} \times \chi \operatorname{curl} \tilde{\mathbf{u}}_{h}\right]_{J}, \mathbf{j}_{h} \lambda_{F}\right\rangle \mathrm{d} \sigma .
$$

Green's formula applied on (34) yields

$$
\begin{aligned}
\frac{c \chi_{A}\left(\eta_{1}^{F}\right)^{2}}{h_{F}} & \leq \int_{T_{1} \cup T_{2}}\left(\left\langle-\operatorname{curl} \chi \operatorname{curl} \tilde{\mathbf{u}}_{h}, \mathbf{j}_{h} \lambda_{F}\right\rangle+\left\langle\chi \operatorname{curl} \tilde{\mathbf{u}}_{h}, \operatorname{curl}\left(\lambda_{F} \mathbf{j}_{h}\right)\right\rangle\right) \mathrm{d} \mathbf{x} \\
& =-r\left(\lambda_{F} \mathbf{j}_{h}\right)+\left(\mathbf{f}-\beta \tilde{\mathbf{u}}_{h}-\operatorname{curl}\left(\chi \operatorname{curl} \tilde{\mathbf{u}}_{h}, \lambda_{F} \mathbf{j}_{h}\right)_{\boldsymbol{L}^{2}\left(T_{1} \cup T_{2}\right)}\right. \\
& \leq C\|\mathbf{e}\|_{\mathfrak{E} ; T_{1} \cup T_{2}}\left\|\lambda_{T} \mathbf{j}_{h}\right\|_{\mathfrak{E} ; T_{1} \cup T_{2}}+C\left\|\sqrt{\chi} \lambda_{T} \mathbf{j}_{h}\right\|_{\boldsymbol{L}^{2}\left(T_{1} \cup T_{2}\right)}\left(h_{T_{1}}^{-1}\left(\eta_{1 ; 1}^{T_{1}}+\eta_{1 ; 2}^{T_{1}}\right)+h_{T_{2}}^{-1}\left(\eta_{1 ; 1}^{T_{2}}+\eta_{1 ; 2}^{T_{2}}\right)\right) \\
& \leq C\left(h_{T_{1}}^{-1}\left(\|\mathbf{e}\|_{\mathfrak{E} ; T_{1}}+\eta_{1 ; 1}^{T_{1}}+\eta_{1 ; 2}^{T_{1}}\right)+h_{T_{2}}^{-1}\left(\left(\|\mathbf{e}\|_{\mathfrak{E} ; T_{2}}+\eta_{1 ; 1}^{T_{2}}+\eta_{1 ; 2}^{T_{2}}\right)\right) \chi_{A} \eta_{1}^{F} .\right.
\end{aligned}
$$

Combining the bound for $\eta_{1 ; 1}^{T}$ with the last inequality provides an upper bound for $\eta_{1}^{F}$ in terms of the local energy norm of the total error and $\eta_{1 ; 2}^{T_{1}}, \eta_{1 ; 2}^{T_{2}}$

$$
\eta_{1}^{F} \leq C\left(\|\mathbf{e}\|_{\mathfrak{E} ; T_{1}}+\|\mathbf{e}\|_{\mathfrak{E} ; T_{2}}+\eta_{1 ; 2}^{T_{1}}+\eta_{1 ; 2}^{T_{2}}\right) .
$$




\section{NumericAl EXPERIMENTS}

In order to demonstrate the performance of the proposed error estimator for a variety of settings, we provide several numerical examples. Throughout we use lowest order edge elements on an unstructured tetrahedral grid. The stiffness matrix and load vector corresponding to (6) are computed using Gaussian quadrature of order 5. Interpolation of boundary values is of the same order. The linear systems of equations are approximately solved by means of a multigrid preconditioned conjugate gradient method [14,15]. The iterations are terminated, when the Euclidean norm of the algebraic residual for the current iterate is less than $10^{-10}$ times the Euclidean norm of the vector on the right-hand side. Thus, the truncation error $\eta_{\text {it }}$ can be neglected.

In all cases the local error estimator (17) in the form of (18) was used to provide the local and global error estimates. Most of the examples were chosen so that the exact solution and, hence, the energy norm $\eta_{T}$ of the true error were available for each element $T$.

To gauge the quality of the error estimator in particular settings we evaluate different functionals:

- The effectivity index $\varepsilon:=\hat{\eta} / \eta$, which gives the ratio between the estimated and the true discretization error. Here, $\eta:=\|\mathbf{e}\|_{\mathfrak{E} ; \Omega}$ and $\hat{\eta}^{2}:=\sum_{T} \hat{\eta}_{T}^{2}$. This quantity merely reflects the quality of the global estimate. For a good error estimator, the effectivity index is to approach a constant rapidly as refinement proceeds. We point out that, since we can only expect equivalence of the estimated energy of the error and its true energy, the effectivity index may be far off the ideal value 1.

- The proportion $\mu^{(1)}$ of "incorrect decisions", measuring how much refinement controlled by the actual estimator differs from refinement based upon an "ideal" estimator. Consider the set of elements marked for refinement by the error estimator

$$
\hat{A}:=\left\{T \in \mathcal{T}_{h}: \hat{\eta}_{T}^{2}>\sigma \frac{1}{n_{T}} \sum_{T \in \mathcal{T}_{h}} \hat{\eta}_{T}^{2}\right\},
$$

where $\sigma=0.95$ and $n_{T}=\# \mathcal{T}_{h}$, and the set of elements that should have been marked

$$
A:=\left\{T \in \mathcal{T}_{h}: \eta_{T}^{2}>\sigma \frac{1}{n_{T}} \sum_{T \in \mathcal{T}_{h}} \eta_{T}^{2}\right\} .
$$

Then we define

$$
\mu^{(1)}:=\frac{1}{n_{T}} \#\{(A \cap C \hat{A}) \cup(C A \cap \hat{A})\}
$$

If the estimator performs satisfactorily, we expect $\mu^{(1)}$ to stay bounded well below 1 as refinement proceeds.

- A measure $\mu^{(2)}$ for the "severity of incorrect decisions", which gives crude information how much smaller the discretization error might have been, if an "ideal" estimator had steered local refinement. Since lowest order edge elements provide a first order approximation in the energy norm, we expect a reduction of the error on a single element by local refinement roughly like $\eta_{T}^{2} \rightarrow \frac{1}{4} \eta_{T}^{2}$. Thus the total error on the adaptively refined mesh can be expressed as

$$
\eta_{\text {new }}^{2}=\frac{1}{4} \sum_{T \in \hat{A}} \eta_{T}^{2}+\sum_{T \in C \hat{A}} \eta_{T}^{2}
$$

If $A$ is substituted for $\hat{A}$, a case we regard as "optimal" local refinement, we end up with an error $\eta_{\text {opt }}^{2}$.

$$
\mu^{(2)}:=\frac{\left|\eta_{\mathrm{new}}^{2}-\eta_{\mathrm{opt}}^{2}\right|}{\eta_{\mathrm{opt}}^{2}}
$$


TABLE 1. Quality measures for the residual based error estimator on the unit cube (Exp. 1).

\begin{tabular}{|l|cccccc|}
\hline \multicolumn{1}{|c|}{ Level } & 0 & 1 & 2 & 3 & 4 & 5 \\
\hline \multicolumn{7}{|c|}{ Effectivity index $\epsilon$} \\
\hline \hline$\beta=10^{-4}$ & 4.05 & 8.05 & 8.18 & 8.24 & 8.27 & 8.29 \\
$\beta=10^{-2}$ & 4.05 & 8.05 & 8.17 & 8.23 & 8.27 & 8.29 \\
$\beta=1.0$ & 3.01 & 7.64 & 7.78 & 7.84 & 7.87 & 7.89 \\
$\beta=10^{2}$ & 2.29 & 4.27 & 4.70 & 4.95 & 5.20 & 5.26 \\
$\beta=10^{4}$ & 2.33 & 4.23 & 4.66 & 4.86 & 4.95 & 5.00 \\
\hline \hline$\mu^{(1)}$ & \multicolumn{7}{c}{} \\
\hline \hline$\beta=10^{-4}$ & 0.33 & 0.17 & 0.12 & 0.1 & 0.1 & 0.085 \\
$\beta=10^{-2}$ & 0.33 & 0.17 & 0.12 & 0.1 & 0.1 & 0.086 \\
$\beta=1.0$ & 0.33 & 0.25 & 0.18 & 0.14 & 0.13 & 0.13 \\
$\beta=10^{2}$ & 0 & 0.42 & 0.088 & 0.14 & 0.15 & 0.16 \\
$\beta=10^{4}$ & 0 & 0.44 & 0.11 & 0.15 & 0.16 & 0.16 \\
\hline \hline$\mu^{(2)}$ & \multicolumn{7}{c}{} \\
\hline \hline$\beta=10^{-4}$ & 0.28 & 0.41 & 0.057 & 0.033 & 0.014 & 0.0062 \\
$\beta=10^{-2}$ & 0.28 & 0.41 & 0.056 & 0.033 & 0.014 & 0.0062 \\
$\beta=1.0$ & 0.29 & 0.056 & 0.0039 & 0.013 & 0.034 & 0.038 \\
$\beta=10^{2}$ & 0 & 0.091 & 0.014 & 0.0085 & 0.0039 & 0.0034 \\
$\beta=10^{4}$ & 0 & 0.26 & 0.078 & 0.074 & 0.065 & 0.047 \\
\hline \hline$\mu^{(3)}$ & \multicolumn{7}{c}{} \\
\hline \hline$\beta=10^{-4}$ & 0.2 & 0.078 & 0.026 & 0.0079 & 0.0026 & 0.00089 \\
$\beta=10^{-2}$ & 0.2 & 0.078 & 0.026 & 0.0079 & 0.0026 & 0.00089 \\
$\beta=1.0$ & 0.2 & 0.084 & 0.025 & 0.0077 & 0.0026 & 0.00089 \\
$\beta=10^{2}$ & 0.22 & 0.081 & 0.024 & 0.0086 & 0.0032 & 0.0012 \\
$\beta=10^{4}$ & 0.21 & 0.08 & 0.024 & 0.0082 & 0.0029 & 0.0011 \\
\hline
\end{tabular}

If $\mu^{(2)}$ stays neatly bounded, the error estimator performs satisfactorily.

- To be able to zero in on singularities, the error estimator must detect local errors. We define the quantity $\mu^{(3)}$ by

$$
\mu^{(3)}:=\sqrt{\sum_{T \in \mathcal{T}_{H}}\left(\left|\Omega_{T}\right|\left(\frac{\hat{\eta}_{T}^{2}}{\left\|\hat{\eta}^{2}\right\|_{L^{1}}}-\frac{\eta_{T}^{2}}{\left\|\eta^{2}\right\|_{L^{1}}}\right)\right)^{2}} .
$$

with

$$
\left\|\eta^{2}\right\|_{L^{1}}=\sum_{T \in \mathcal{T}_{H}} \eta_{T}^{2}\left|\Omega_{T}\right|, \quad\left\|\hat{\eta}^{2}\right\|_{L^{1}}=\sum_{T \in \mathcal{T}_{H}} \hat{\eta}_{T}^{2}\left|\Omega_{T}\right|
$$

This quantity will be big, if the estimator fails to tell the approximate spatial distribution of the discretization error.

- The gain from adaptive refinement is illustrated by plotting the discretization error versus the total number of degrees of freedom both for uniform and adaptive refinement.

All numerical experiments are conducted on uniformly refined meshes, some of them also on meshes generated by adaptive refinement. The latter relies on an averaging strategy, which singles out the elements in the set $\hat{A}$ from (35) for refinement. When we do so, we also monitor the decrease of the true error against the degrees of freedom for both uniform and adaptive cases in order to assess the gain of adaptivity. 
TABLE 2. Measures for quality of residual based error estimator for Exp. 2.

\begin{tabular}{|c|c|c|c|c|c|c|}
\hline Level & 0 & 1 & 2 & 3 & 4 & 5 \\
\hline \multicolumn{7}{|c|}{ Effectivity index $\epsilon$} \\
\hline $\bar{\beta}=10^{-4}$ & $\overline{5.26}$ & 5.94 & 7.07 & 7.51 & 7.72 & 7.84 \\
\hline$\beta=10^{-2}$ & 5.25 & 6.23 & 7.08 & 7.50 & 7.71 & 7.84 \\
\hline$\beta=1.0$ & 3.82 & 6.08 & 6.92 & 7.34 & 7.55 & 7.66 \\
\hline$\beta=10^{2}$ & 1.86 & 3.14 & 3.99 & 4.46 & 4.96 & 5.09 \\
\hline$\beta=10^{4}$ & 1.70 & 3.17 & 3.98 & 4.34 & 4.49 & 4.57 \\
\hline \multicolumn{7}{|l|}{$\mu^{(1)}$} \\
\hline $\bar{\beta}=10^{-4}$ & 0.33 & 0.19 & 0.13 & 0.1 & 0.1 & 0.1 \\
\hline$\beta=10^{-2}$ & 0.33 & 0.15 & 0.13 & 0.1 & 0.1 & 0.1 \\
\hline$\beta=1.0$ & 0.5 & 0.17 & 0.14 & 0.1 & 0.1 & 0.1 \\
\hline$\beta=10^{2}$ & 0.83 & 0.42 & 0.25 & 0.17 & 0.17 & 0.17 \\
\hline$\beta=10^{4}$ & 0.83 & 0.4 & 0.25 & 0.16 & 0.13 & 0.12 \\
\hline \multicolumn{7}{|l|}{$\mu^{(2)}$} \\
\hline$\beta=10^{-4}$ & 0.31 & 0.043 & 0.054 & 0.037 & 0.041 & 0.044 \\
\hline$\beta=10^{-2}$ & 0.31 & 0.062 & 0.059 & 0.036 & 0.041 & 0.044 \\
\hline$\beta=1.0$ & 0.055 & 0.084 & 0.048 & 0.029 & 0.039 & 0.042 \\
\hline$\beta=10^{2}$ & 0.74 & 0.39 & 0.11 & 0.068 & 0.015 & 0.01 \\
\hline$\beta=10^{4}$ & 0.9 & 0.36 & 0.18 & 0.1 & 0.069 & 0.057 \\
\hline \multicolumn{7}{|l|}{$\mu^{(3)}$} \\
\hline$\beta=10^{-4}$ & 0.1 & 0.06 & 0.033 & 0.013 & 0.0044 & 0.0015 \\
\hline$\beta=10^{-2}$ & 0.1 & 0.065 & 0.033 & 0.013 & 0.0044 & 0.0015 \\
\hline$\beta=1.0$ & 0.12 & 0.065 & 0.032 & 0.012 & 0.0043 & 0.0015 \\
\hline$\beta=10^{2}$ & 0.32 & 0.11 & 0.037 & 0.01 & 0.0032 & 0.001 \\
\hline$\beta=10^{4}$ & 0.35 & 0.12 & 0.04 & 0.011 & 0.0034 & 0.001 \\
\hline
\end{tabular}

The numerical experiments 1 to 5 are carried out on the unit cube $\Omega:=] 0,1\left[{ }^{3}\right.$. Dirichlet boundary conditions are applied on $\partial \Omega$. In order to be able to evaluate the true discretization errors, we choose boundary data and right-hand sides such that an analytical expressions for the solutions of the continuous problems are available. In each case we start with a coarse grid (level 0) consisting of 6 tetrahedrons, which is refined uniformly up to level 5 .

In our first experiment the coefficients $\alpha$ and $\beta$ are kept constant all over the domain; $\chi$ is always set to 1 . In this situation the regularity Assumption 2 is fulfilled. Different values for $\beta$ are taken into account, because in the case of implicit timestepping $\beta$ will be scaled by the size of the timestep. Therefore, it is essential that the error estimator is robust with respect to the relative scaling of $\chi$ and $\beta$. The solution is rather smooth and is given by $\mathbf{u}=\left(0,0, \sin \left(\pi x_{1}\right)\right)$. Consequently there are no particular local features to be detected.

The results are reported in Table 1 and bear out a decent performance of the estimator for this benign setting. We also observe that the error estimator is not severely affected by different values for $\beta$.

For our second experiment boundary data and right-hand sides are chosen such that the solenoidal solution $\mathbf{u}=\operatorname{curl}\left(\sin \left(\pi x_{2} x_{3}\right), \cos \left(\pi x_{1} x_{3}\right), \sin \left(\pi x_{1} x_{2}\right)\right)$ is generated. We included this experiment to study how the error estimator responds to a smooth divergence-free solution.

We refer to Table 2 for the results. Little difference compared to the previous experiment can be seen.

In the third experiment we generate a smooth irrotational solution $\mathbf{u}=\operatorname{grad}(x y z)$. The coefficients $\chi$ and $\beta$ are chosen like in the previous experiments. In this case the irrotational part of the error is the only remaining component. 
TABLE 3. Measures for the quality of the residual based error estimator on the unit cube (Exp. 3).

\begin{tabular}{|l|cccccc|}
\hline \multicolumn{1}{|c|}{ Level } & 0 & 1 & 2 & 3 & 4 & 5 \\
\hline \hline \multicolumn{7}{|c|}{ Effectivity index $\epsilon$} \\
\hline \hline$\beta=10^{-4}$ & 4.21 & 4.70 & 4.90 & 4.98 & 5.01 & 5.03 \\
$\beta=10^{-2}$ & 4.21 & 4.70 & 4.90 & 4.98 & 5.01 & 5.03 \\
$\beta=1.0$ & 4.21 & 4.70 & 4.89 & 4.98 & 5.01 & 5.03 \\
$\beta=10^{2}$ & 4.22 & 4.68 & 4.87 & 4.96 & 5.01 & 5.03 \\
$\beta=10^{4}$ & 4.22 & 4.66 & 4.84 & 4.92 & 4.96 & 4.99 \\
\hline \hline$\mu^{(1)}$ & \multicolumn{7}{c}{} \\
\hline \hline$\beta=10^{-4}$ & 0.5 & 0.042 & 0.13 & 0.14 & 0.14 & 0.14 \\
$\beta=10^{-2}$ & 0.5 & 0.042 & 0.13 & 0.14 & 0.14 & 0.14 \\
$\beta=1.0$ & 0.5 & 0.042 & 0.13 & 0.14 & 0.14 & 0.14 \\
$\beta=10^{2}$ & 0.33 & 0.1 & 0.13 & 0.14 & 0.14 & 0.14 \\
$\beta=10^{4}$ & 0.33 & 0.12 & 0.14 & 0.13 & 0.14 & 0.14 \\
\hline \hline$\mu^{(2)}$ & \multicolumn{7}{c}{} \\
\hline \hline$\beta=10^{-4}$ & 0.46 & 0.054 & 0.042 & 0.037 & 0.034 & 0.023 \\
$\beta=10^{-2}$ & 0.46 & 0.054 & 0.042 & 0.037 & 0.034 & 0.023 \\
$\beta=1.0$ & 0.46 & 0.054 & 0.047 & 0.037 & 0.034 & 0.023 \\
$\beta=10^{2}$ & 0.32 & 0.057 & 0.04 & 0.036 & 0.032 & 0.023 \\
$\beta=10^{4}$ & 0.32 & 0.083 & 0.051 & 0.026 & 0.019 & 0.016 \\
\hline \hline$\mu^{(3)}$ & \multicolumn{7}{c}{} \\
\hline \hline$\beta=10^{-4}$ & 0.24 & 0.095 & 0.032 & 0.01 & 0.0034 & 0.0011 \\
$\beta=10^{-2}$ & 0.24 & 0.095 & 0.032 & 0.01 & 0.0034 & 0.0011 \\
$\beta=1.0$ & 0.24 & 0.095 & 0.032 & 0.01 & 0.0034 & 0.0011 \\
$\beta=10^{2}$ & 0.24 & 0.082 & 0.03 & 0.0098 & 0.0033 & 0.0011 \\
$\beta=10^{4}$ & 0.24 & 0.077 & 0.028 & 0.0088 & 0.003 & 0.001 \\
\hline
\end{tabular}

Values for the different quality measures are given in Table 3 . They show that the error estimator is insensitive to irrotational solutions.

Our fourth experiment deals with constant $\beta=1$, whereas the other coefficient is varying on the domain: $\chi(\mathbf{x})=1.5+\sin \left(2 \pi x_{1}\right) \sin \left(2 \pi x_{2}\right) \sin \left(2 \pi x_{3}\right)$. We choose the smooth solution $\boldsymbol{\xi}=\left(0,0, \sin \left(\pi x_{1}\right)\right)$. In this case the energy norm features a certain anisotropy, but the coefficients are still smooth.

How the error estimator behaves can be seen from Table 4. All the observations made in the previous experiments remain true.

TABLE 4. Measures for the quality of the residual based error estimator on the unit cube (Exp. 4).

\begin{tabular}{|l|cccccc|}
\hline Level & 0 & 1 & 2 & 3 & 4 & 5 \\
\hline \hline$\epsilon$ & 5.59 & 9.80 & 11.20 & 11.46 & 11.57 & 11.61 \\
\hline$\mu^{(1)}$ & 0.67 & 0.31 & 0.21 & 0.2 & 0.19 & 0.19 \\
\hline$\mu^{(2)}$ & 0.32 & 0.28 & 0.072 & 0.1 & 0.11 & 0.11 \\
\hline$\mu^{(3)}$ & 0.26 & 0.077 & 0.028 & 0.0095 & 0.0033 & 0.0011 \\
\hline
\end{tabular}

The fifth experiment exchanges the roles of the coefficients. Now $\chi$ is set to 1 throughout the domain and $\beta$ is given by $\beta(\mathbf{x})=1.5+\sin \left(2 \pi x_{1}\right) \sin \left(2 \pi x_{2}\right) \sin \left(2 \pi x_{3}\right)$. Again the solution is $\mathbf{u}=\left(0,0, \sin \left(\pi x_{1}\right)\right)$.

See Table 5 for information about the performance of the error estimator. We remark that the performance remains satisfactory. 
TABLE 5. Measures for the quality of the residual based error estimator on the unit cube (Exp. 5).

\begin{tabular}{|l|cccccc|}
\hline Level & 0 & 1 & 2 & 3 & 4 & 5 \\
\hline \hline$\epsilon$ & 2.92 & 7.83 & 8.18 & 8.97 & 11.47 & 18.37 \\
\hline$\mu^{(1)}$ & 0.33 & 0.35 & 0.19 & 0.18 & 0.17 & 0.18 \\
\hline$\mu^{(2)}$ & 0.29 & 0.071 & 0.0027 & 0.025 & 0.083 & 0.15 \\
\hline$\mu^{(3)}$ & 0.22 & 0.085 & 0.025 & 0.0082 & 0.0032 & 0.0014 \\
\hline
\end{tabular}

Our sixth experiment is again carried out on the unit cube $\Omega:=] 0,1\left[{ }^{3}\right.$ with $\chi \equiv 1$, but we enforce a vanishing zero-order term on part of the domain. Thus we depart from the situation where the regularity assumption holds. As far as the coefficients are concerned, this experiment comes fairly close to the arrangements in realistic eddy current computations.

In particular, we choose $\beta$ as follows:

$$
\beta(\mathbf{x})= \begin{cases}1 & : \max \left\{\left|x_{1}-0.5\right|,\left|x_{2}-0.5\right|,\left|x_{3}-0.5\right|\right\} \leq 0.25 \\ 0 & : \text { elsewhere. }\end{cases}
$$

Boundary data and right-hand side are again adjusted to produce the smooth solution $\mathbf{u}=\left(0,0, \sin \left(\pi x_{1}\right)\right)$. Of course, in this respect we fail to capture the usual singular behavior of the electric field at the edges of the conductor.

The results of the computations are recorded in Table 6. All measures for the quality of the error estimator reveal a flawless performance.

TABLE 6. Measures for the quality of the residual based error estimator on the unit cube (Exp. 6).

\begin{tabular}{|l|ccccc|}
\hline Level & 0 & 1 & 2 & 3 & 4 \\
\hline \hline$\epsilon$ & 5.18 & 7.22 & 7.46 & 7.52 & 7.53 \\
\hline$\mu^{(1)}$ & 0.17 & 0.081 & 0.066 & 0.051 & 0.047 \\
\hline$\mu^{(2)}$ & 0.099 & 0.027 & 0.011 & 0.0026 & 0.0031 \\
\hline$\mu^{(3)}$ & 0.12 & 0.05 & 0.017 & 0.0058 & 0.0021 \\
\hline
\end{tabular}

Owing to the smooth solution adaptive refinement does not really pay off in this situation (cf. Fig 2). Nevertheless, we report the quality measures in Table 7 to show that non-uniform meshes do not make a difference.

TABLE 7. Measures for the quality of the residual based error estimator on the unit cube in the adaptive case (Exp. 6).

\begin{tabular}{|l|ccccccc|}
\hline Level & 0 & 1 & 2 & 3 & 4 & 5 & 6 \\
\hline \hline$\epsilon$ & 5.18 & 7.29 & 7.89 & 7.89 & 7.81 & 7.73 & 7.88 \\
\hline$\mu^{(1)}$ & 0.17 & 0.11 & 0.15 & 0.17 & 0.14 & 0.11 & 0.17 \\
\hline$\mu^{(2)}$ & 0.099 & 0.083 & 0.024 & 0.1 & 0.11 & 0.039 & 0.076 \\
\hline$\mu^{(3)}$ & 0.12 & 0.048 & 0.032 & 0.014 & 0.0054 & 0.0036 & 0.0022 \\
\hline
\end{tabular}

For the seventh experiment we employ the domain and coefficients of the previous one. But now we enforce homogeneous Dirichlet boundary conditions $\mathbf{u} \times \mathbf{n}=0$ on the boundary and a smooth right hand side $\mathbf{f}=(1,1,1)$. 


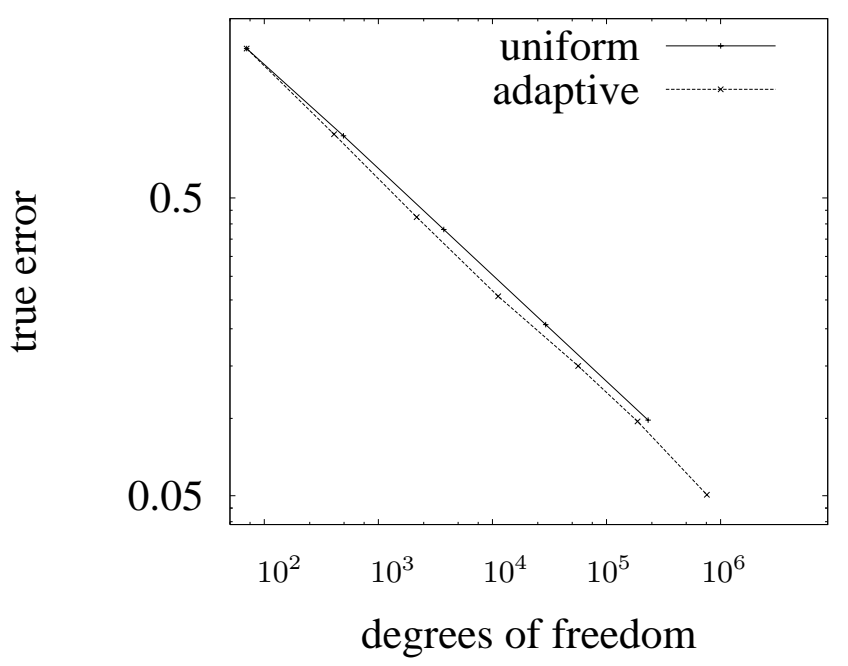

Figure 2. True error for uniform and adaptive mesh refinement on the unit cube (Exp. 6).

TABLE 8. Measures for the quality of the residual based error estimator on the unit cube (Exp. 7).

\begin{tabular}{|l|cccc|}
\hline Level & 0 & 1 & 2 & 3 \\
\hline \hline$\epsilon$ & 15.19 & 10.70 & 9.89 & 10.34 \\
\hline$\mu^{(1)}$ & 0.1 & 0.094 & 0.093 & 0.1 \\
\hline$\mu^{(2)}$ & 0.24 & 0.016 & 0.051 & 0.079 \\
\hline$\mu^{(3)}$ & 0.076 & 0.046 & 0.021 & 0.0083 \\
\hline
\end{tabular}

TABLE 9. Measures for the quality of the residual based error estimator on the unit cube in the adaptive case (Exp. 7).

\begin{tabular}{|l|cccccc|}
\hline Level & 0 & 1 & 2 & 3 & 4 & 5 \\
\hline \hline$\epsilon$ & 15.15 & 11.03 & 10.41 & 10.81 & 10.52 & 11.86 \\
\hline$\mu^{(1)}$ & 0.1 & 0.29 & 0.22 & 0.12 & 0.18 & 0.14 \\
\hline$\mu^{(2)}$ & 0.24 & 0.046 & 0.23 & 0.072 & 0.079 & 0.14 \\
\hline$\mu^{(3)}$ & 0.076 & 0.04 & 0.024 & 0.015 & 0.0075 & 0.0041 \\
\hline
\end{tabular}

Thus the vector field will be non-smooth and cannot be described analytically. Now, all the features of an actual eddy current problem are present.

To estimate the true errors, we carried out two refinement steps more than reported in Tables 8 and 9, respectively, and compare the discrete solutions to those obtained on the finest levels. The results are collected in Table 8 for uniform refinement and in Table 9 for adaptive refinement. Evidently, the additional singularities in the solution hardly affect the error estimator in either case. 
TABLE 10. Measures for the quality of the residual based error estimator on the L-shaped domain (Exp. 8).

\begin{tabular}{|l|ccccc|}
\hline Level & 0 & 1 & 2 & 3 & 4 \\
\hline \hline$\epsilon$ & 3.39 & 3.73 & 3.85 & 3.94 & 3.99 \\
\hline$\mu^{(1)}$ & 0.27 & 0.1 & 0.047 & 0.029 & 0.019 \\
\hline$\mu^{(2)}$ & 0.17 & 0.079 & 0.07 & 0.018 & 0.013 \\
\hline$\mu^{(3)}$ & 0.11 & 0.07 & 0.047 & 0.032 & 0.022 \\
\hline
\end{tabular}

TABLE 11. Measures for the quality of the residual based error estimator on the L-shaped domain in the adaptive case (Exp. 8).

\begin{tabular}{|l|ccccccc|}
\hline Level & 0 & 1 & 2 & 3 & 4 & 5 & 6 \\
\hline \hline$\epsilon$ & 3.39 & 3.67 & 3.82 & 4.05 & 4.18 & 4.28 & 4.37 \\
\hline$\mu^{(1)}$ & 0.27 & 0.18 & 0.12 & 0.12 & 0.11 & 0.096 & 0.088 \\
\hline$\mu^{(2)}$ & 0.17 & 0.11 & 0.08 & 0.018 & 0.012 & 0.021 & 0.025 \\
\hline$\mu^{(3)}$ & 0.11 & 0.065 & 0.11 & 0.037 & 0.02 & 0.0056 & 0.0027 \\
\hline
\end{tabular}

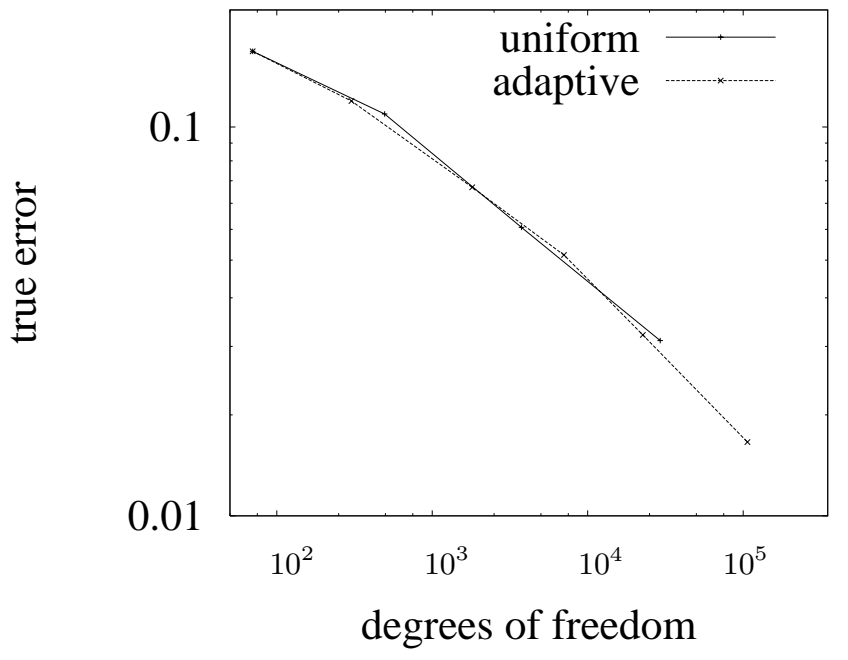

Figure 3. True error for uniform and adaptive mesh refinement on the unit cube (Exp. 7).

Surprisingly, the singularities do hardly reward adaptive refinement, as can be seen from Figure 3. However, as the right tails of the curves indicate, adaptivity might pay off on higher levels. But due to lacking computer resources we could not proceed with grid refinement in our experiment.

The eighth experiment deals with an edge singularity of the field. We use a non-convex "L-shaped" domain $\Omega:=]-1,1\left[^{3} \backslash[0,1]^{2} \times[-1,1]\right.$. The coarsest grid comprises 52 tetrahedra. 


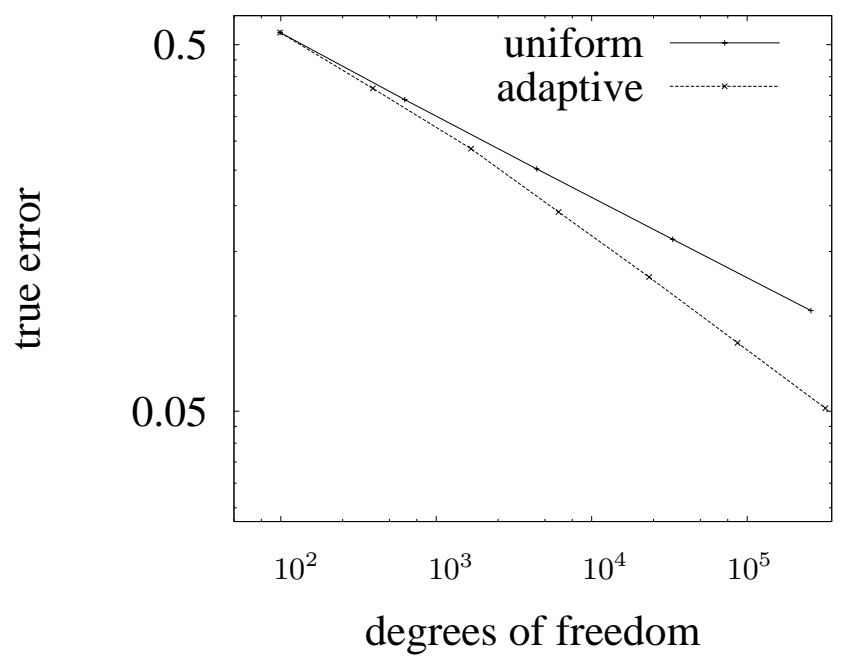

Figure 4. True error for uniform and adaptive mesh refinement on the L-shaped domain (Exp. 8).
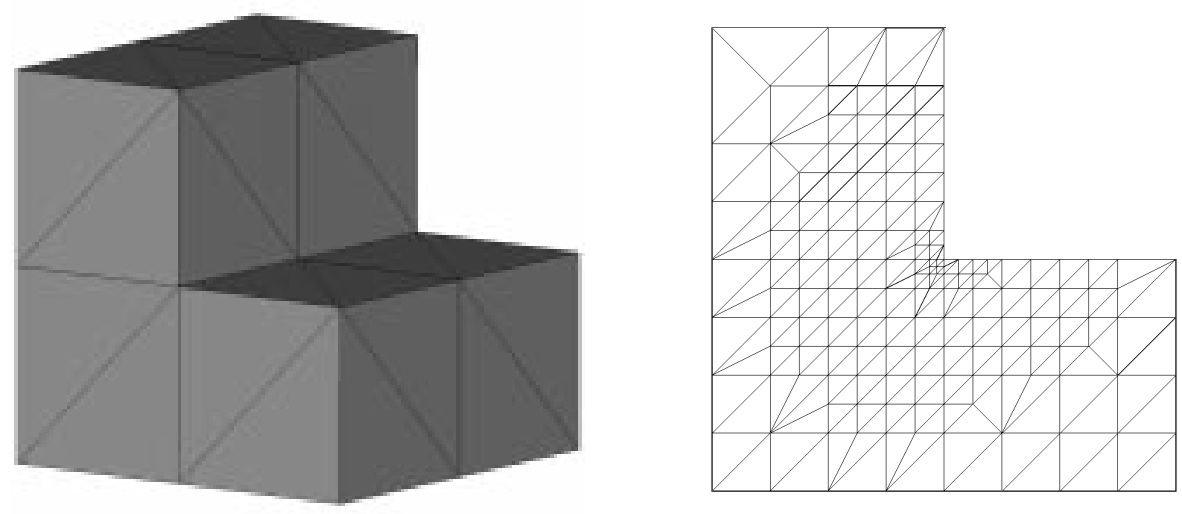

Figure 5. The L-shaped domain of Exp. 8. On the left-hand side the initial triangulation is shown; the other figure displays a cross section of the grid after five adaptive refinement steps.

We set $\chi$ and $\beta$ to 1 and employ such boundary conditions and right-hand side that the solution is given (in polar coordinates $)$ by $\mathbf{u}=\operatorname{grad}\left(r^{\frac{2}{3}} \sin \left(\frac{2}{3} \phi\right)\right.$. The gradient field $\mathbf{u}$ is both irrotational and divergence-free and does not even belong to $\boldsymbol{H}^{1}(\Omega)$.

How this affects the error estimator is conveyed by Table 10 for the case of uniform refinement and Table 11 for local grid adaptation. The numbers illustrate that the error estimator can be relied upon even under these extreme circumstances. 
Now that the vectorfield is singular along the concave edge of the L-shaped domain, local mesh refinement should provide favourable grids. Indeed, Fig. 4 reveals a clearly superior performance in the adaptive case. Figure 5 gives a view of both the initial and an adaptively refined triangulation; observe how the grid adaption concentrates on the concave edge of the domain.

\section{Conclusion}

In the present paper we have designed a local a posteriori error estimator for $\boldsymbol{H}(\mathbf{c u r l} ; \Omega)$-elliptic problems by taking into account the dual norm of the residual. We could show that this estimator is efficient, i.e. apart from scaling it provides a local lower bound for the energy norm of the error. Under additional assumptions we could establish that it is also reliable in the sense that we can also obtain a global upper bound. However, the assumptions are hardly ever met in realistic settings. Nevertheless, the numerical results offer strong evidence that the error estimator performs excellently beyond the scope of the theoretical analysis. Hence, it would be desirable to extend the rigorous treatment to the case of discontinuous coefficients.

\section{REFERENCES}

[1] B. Achchab, A. Agouzal, J. Baranger and J. Maitre, Estimateur d'erreur a posteriori hiérarchique. Application aux éléments finis mixtes. IMPACT Comput. Sci. Engrg. 1 (1995) 3-35.

[2] R. Albanese and G. Rubinacci, Formulation of the eddy-current problem. IEE Proc. A 137 (1990) 16-22.

[3] Analysis of three dimensional electromagnetic fileds using edge elements. J. Comp. Phys. 108 (1993) 236-245.

[4] A. Alonso and A. Valli, Some remarks on the characterization of the space of tangential traces of $H(\operatorname{rot} ; \Omega)$ and the construction of an extension operator. Manuscripta math. 89 (1996) 159-178.

[5] H. Ammari, A. Buffa and J.-C. Nédélec, A justification of eddy currents model for the Maxwell equations. Tech. Rep., IAN, University of Pavia, Pavia, Italy (1998).

[6] C. Amrouche, C. Bernardi, M. Dauge and V. Girault, Vector potentials in three-dimensional nonsmooth domains. Math. Methods Appl. Sci. 21 (1998) 823-864.

[7] D. Arnold, A. Mukherjee and L. Pouly, Locally adapted tetrahedral meshes using bisection. SIAM J. on Sci. Compt (submitted).

[8] I. Babuška and W. Rheinboldt, Error estimates for adaptive finite element computations, SIAM J. Numer. Anal. 15 (1978) $736-754$.

[9] I. Babuška and W. Rheinboldt, A posteriori error estimates for the finite element method. Internet. J. Numer. Methods Engrg. 12 (1978) 1597-1615.

[10] R. Bank, PLTMG: A Software Package for Solving Elliptic Partial Differential Equations, User's Guide 6.0. SIAM, Philadelphia (1990).

[11] R. Bank, A. Sherman and A. Weiser, Refinement algorithm and data structures for regular local mesh refinement., in Scientific Computing, R. Stepleman et al., Ed., Vol. 44, IMACS North-Holland, Amsterdam (1983) 3-17.

[12] R. Bank and A. Weiser, some a posteriori error estimators for elliptic partial differential equations. Math. Comp. 44 (1985) 283-301.

[13] E. Bänsch, Local mesh refinement in 2 and 3 dimensions. IMPACT Comput. Sci. Engrg. 3 (1991) 181-191.

[14] R. Beck, P. Deuflhard, R. Hiptmair, R. Hoppe and B. Wohlmuth, Adaptive multilevel methods for edge element discretizations of Maxwell's equations. Surveys for Mathematics in Industry.

[15] R. Beck and R. Hiptmair, Multilevel solution of the time-harmonic Maxwell equations based on edge elements. Tech. Rep. SC-96-51, ZIB Berlin (1996). in Internat. J. Numer. Methods Engrg. (To appear).

[16] J. Bey, Tetrahedral grid refinement. Computing 55 (1995) 355-378.

[17] F. Bornemann, An adaptive multilevel approach to parabolic equations I. General theory and 1D-implementation. IMPACT Comput. Sci. Engrg. 2 (1990) 279-317.

[18] F. Bornemann, An adaptive multilevel approach to parabolic equations II. Variable-order time discretization based on a multiplicative error correction. IMPACT Comput. Sci. Engrg. 3 (1991) 93-122.

[19] F. Bornemann, B. Erdmann and R. Kornhuber, A posteriori error estimates for elliptic problems in two and three spaces dimensions. SIAM J. Numer. Anal. 33 (1996) 1188-1204.

[20] A. Bossavit, Mixed finite elements and the complex of Whitney forms, in The Mathematics of Finite Elements and Applications VI J. Whiteman Ed., Academic Press, London (1988) 137-144.

[21] A. Bossavit, A rationale for edge elements in 3D field computations. IEEE Trans. Mag. 24 (1988) 74-79.

[22] A. Bossavit, Solving Maxwell's equations in a closed cavity and the question of spurious modes. IEEE Trans. Mag. 26 (1990) $702-705$.

[23] A. Bossavit, Électromagnétisme, en vue de la modélisation. Springer-Verlag, Paris (1993). 
[24] A. Bossavit, Computational Electromagnetism. Variational Formulation, Complementarity, Edge Elements.in Academic Press Electromagnetism Series, no. 2 Academic Press, San Diego (1998).

[25] D. Braess and R. Verfürth, A posteriori error estimators for the Raviart-Thomas element. SIAM J. Numer. Anal. 33 (1996) 2431-2445.

[26] C. Carstensen, A posteriori error estimate for the mixed finite element method. Math. Comp. 66 (1997) 465-476.

[27] P. Ciarlet, The Finite Element Method for Elliptic Problems. Studies in Mathematics and its Applications, Vol. 4 NorthHolland, Amsterdam (1978).

[28] M. Clemens, R. Schuhmann, U. van Rienen and T. Weiland, Modern Krylov subspace methods in electromagnetic field computation using the finite integration theory. ACES J. Appl. Math. 11 (1996) 70-84.

[29] M. Clemens and T. Weiland, Transient eddy current calculation with the FI-method. in Proc. CEFC '98, IEEE (1998); IEEE Trans. Mag. submitted

[30] P. Clément, Approximation by finite element functions using local regularization. Revue Franc. Automat. Inform. Rech. Operat. 9, R-2 (1975) 77-84.

[31] M. Costabel and M. Dauge, Singularities of electromagnetic fields in polyhedral domains. Tech. Rep. 97-19, IRMAR, Rennes, France (1997)

[32] M. Costabel, M. Dauge and S. Nicaise, Singularities of Maxwell interface problems, Tech. Rep. 98-24, IRMAR, Rennes, France (1998).

[33] H. Dirks, Quasi-stationary fields for microelectronic applications. Electrical Engineering 79 (1996) 145-155.

[34] P. Dular, J.-Y. Hody, A. Nicolet, A. Genon and W. Legros, Mixed finite elements associated with a collection of tetrahedra, hexahedra and prisms. IEEE Trans Magnetics MAG-30 (1994) 2980-2983.

[35] K. Erikson, D. Estep, P. Hansbo and C. Johnson, Introduction to adaptive methods for differential equations. Acta Numerica 4 (1995) 105-158.

[36] K. Eriksson and C. Johnson, An adaptive finite element method for linear elliptic problems. Math. Comp. 50 (1988) 361-383.

[37] V. Girault and P. Raviart, Finite element methods for Navier-Stokes equations, Springer-Verlag, Berlin (1986).

[38] E. Hairer and G. Wanner, Solving Ordinary Differential Equations II. Stiff and Differential-Algebraic Problems. SpringerVerlag, Berlin, Heidelberg, New York (1991).

[39] R. Hiptmair, Multigrid method for Maxwell's equations. Tech. Rep. 374, Institut für Mathematik, Universität Augsburg (1997).

[40] R. Hiptmair, Canonical construction of finite elements. Math. Comp. 68 (1999) 1325-1346.

[41] R. Hoppe and B. Wohlmuth, Adaptive multilevel iterative techniques for nonconforming finite element discretizations. EastWest J. Numer. Math. 3 (1995) 179-197.

[42] R. Hoppe and B. Wohlmuth, A comparison of a posteriori error estimators for mixed finite elements. Math. Comp. 68 (1999) $1347-1378$.

[43] R. Hoppe and B. Wohlmuth, Element-oriented and edge-oriented local error estimators for nonconforming finite element methods. Model. Math. Anal. Numér. 30 (1996) 237-263.

[44] R. Hoppe and B. Wohlmuth, Adaptive multilevel techniques for mixed finite element discretizations of elliptic boundary value problems. SIAM J. Numer. Anal. 34 (1997) 1658-1687.

[45] R. Hoppe and B. Wohlmuth, Hierarchical basis error estimators for Raviart-Thomas discretizations of arbitrary order, in Finite Element Methods: Superconvergence, Post-processing and A Posteriori Estimates, M. Krizck, P. Neittaanmäki and R. Stenberg Eds., Marcel Dekker, New York (1997) 155-167.

[46] J. Maubach, Local bisection refinement for $n$-simplicial grids generated by reflection. SIAM J. Sci. Stat. Comp. 16 (1995) 210-227.

[47] P. Monk, A mixed method for approximating Maxwell's equations. SIAM J. Numer. Anal. 28 (1991) 1610-1634.

[48] P. Monk, Analysis of a finite element method for Maxwell's equations. SIAM J. Numer. Anal. 29 (1992) 714-729.

[49] J. Nédélec, Mixed finite elements in $R^{3}$, Numer. Math. 35 (1980) 315-341.

[50] E. Ong, Hierarchical basis preconditioners for second order elliptic problems in three dimensions. Ph.D. thesis, Dept. of Math., UCLA, Los Angeles, CA, USA (1990).

[51] P. Oswald, Multilevel finite element approximation. Teubner Skripten zur Numerik, B.G. Teubner, Stuttgart (1994).

[52] J. P. Ciarlet and J. Zou, Fully discrete finite element approaches for time-dependent Maxwell equations. Tech. Rep. TR MATH-96-31 (105), Department of Mathematics, The Chinese University of Hong Kong (1996). Num. Math. (to appear).

[53] L. R. Scott and Z. Zhang, Finite element interpolation of nonsmooth functions satisfying boundary conditions. Math. Comp. 54 (1990) 483-493.

[54] R. Verfürth, A posteriori error estimators for nonlinear problems. Finite element discretizations of elliptic equations. Math. Comp. 62 (1994) 445-475.

[55] R. Verfürth, A Review of A Posteriori Error Estimation and Adaptive Mesh-Refinement Techniques. Wiley-Teubner, Chichester, Stuttgart (1996).

[56] H. Whitney, Geometric Integration Theory. Princeton Univ. Press, Princeton (1957).

[57] J. Zhu and O. Zienkiewicz, Adaptive techniques in the finite element method. Commun. Appl. Numer. Methods 4 (1988) 197-204. 